\title{
Near-IR and visible interferometry of Be stars: Constraints from wind models
}

\author{
Ph. Stee ${ }^{1}$ and J. Bittar ${ }^{2}$ \\ 1 Observatoire de la Côte d'Azur, Département Fresnel UMR 6528, Caussols, 06460 St. Vallier de Thiey, France \\ 2 Observatoire Midi-Pyrénées, CNRS UMR 5572, 14 Av. Edouard Belin, 31400 Toulouse, France
}

Received 5 October 2000 / Accepted 14 November 2000

\begin{abstract}
We report theoretical $\mathrm{HI}$ visible and near-IR line profiles, i.e. $\mathrm{H} \alpha(6562 \AA), \mathrm{H} \beta(4861 \AA)$ and $\mathrm{Br} \gamma$ $(21656 \AA)$, and intensity maps for a large set of parameters (density, temperature, envelope geometry, inclination angle), representative of early to late Be spectral types. We have computed the size of the emitting region in the $\mathrm{Br} \gamma$ line and its nearby continuum which both originate from a very extended region, i.e. at least 40 stellar radii which is twice the size of the $\mathrm{H} \alpha$ emitting region. We predict the relative fluxes from the central star, the envelope contribution in the given lines and in the continuum for a wide range of parameters characterizing the disk models. For a density $\rho=510^{-13} \mathrm{~g} \mathrm{~cm}^{-3}$ at the base of the stellar photosphere, we obtain the largest probability of HI IR lines in emission, which is a factor of 100 lower than typical values found for Be stars. We have also studied the effect of changing the spectral type on our results and we obtain a clear correlation between the luminosity in $\mathrm{H} \alpha$ and in the infrared. We found that for a density $\rho=510^{-12} \mathrm{~g} \mathrm{~cm}^{-3}$, the probability of detecting HI IR lines in emission must be stronger for late-B spectral type stars. If no IR lines are detected for late types, it may indicate that the density in the disc is very high $\left(\sim 10^{-11} \mathrm{~g} \mathrm{~cm}^{-3}\right)$. On the other hand, we found that around $\rho=$ $510^{-13} \mathrm{~g} \mathrm{~cm}^{-3}$, it is possible to have a large envelope contribution in the Br $\gamma$ line and a similar or even smaller emission in the Balmer lines. Even if $\mathrm{Br} \gamma$ is formed in an extended region, it is possible to obtain a FWHM and a $\mathrm{V} / \mathrm{R}$ that agree well with observed profiles. Finally, it seems that the contribution in the $\operatorname{Br} \gamma$ line increases when the envelope becomes more and more "disk-like", contrary to the $\mathrm{H} \alpha$ and $\mathrm{H} \beta$ lines.
\end{abstract}

Key words. stars: circumstellar matter; emission line; Be - infrared: stars - winds: models - techniques: interferometric

\section{Introduction}

Interferometry in the visible has been used successfully to study the circumstellar environment of Be stars (Thom et al. 1986; Quirrenbach et al. 1993; Stee et al. 1995 (hereafter Paper I), Quirrenbach et al. 1997; see also the recent review given by Stee 2000). It is now clear that the matter responsible for the visible emission lines and the continuum is not spherically distributed. Moreover, in the paper by Stee et al. (1998), we found that the envelope size in the visible increases following the sequence He I $\lambda 6678$ $\left(2.3 R_{*}\right), 0.48 \mu \mathrm{m}$ continuum $\left(2.8 R_{*}\right), 0.65 \mu \mathrm{m}$ continuum $\left(3.5 R_{*}\right), \mathrm{H} \beta\left(\$ 8.5 R_{*}\right)$ and $\mathrm{H} \alpha\left(18 R_{*}\right)$ emitting regions. In fact, the envelope size in the He I $\lambda 6678$ line was under-estimated due to the subtraction of the nearby continuum in our computation. Since the line is formed on its continuum, it was not correct to separate photons from the line and the continuum in the simulation. Adding the

Send offprint requests to: $\mathrm{Ph}$. Stee,

e-mail: Philippe.Stee@obs-azur.fr
$0.65 \mu \mathrm{m}$ continuum, we obtain a He I $\lambda 6678$ emitting region with an angular size of $\sim 4 R_{*}$, in agreement with the size of $3.7 R_{*}$ for the $0.65 \mu \mathrm{m}$ continuum obtained in this paper. In this paper, we extend previous model to nearIR wavelengths, a timely contribution in the context of recent IR spectroscopic studies (Marlborough et al. 1997; Clark \& Steele 2000; Hony et al. 2000) and planned interferometric observations (Richichi et al. 2000; Weigelt et al. 2000). We stress in this work the importance of simultaneous high spectral and angular resolution studies in the visible and near-IR in providing strong constraints on the physics of the circumstellar envelopes of Be stars.

The paper is organized as follows. In Sect. 2 we briefly present the SIMECA code and its main assumptions. Section 3 presents theoretical $\mathrm{HI}$ visible $\mathrm{H} \alpha, \mathrm{H} \beta$, and nearIR $\operatorname{Br} \gamma$ line profiles, as well as computed energy distribution and intensity maps obtained with SIMECA for the particular case of $\gamma$ Cas. In Sect. 4 we study the possibility of extending these results to other Be stars with different wind densities. Section 5 discusses the effect of changing 
the effective temperature of the central star. In Sect. 6 we present results for different spectral types: B0, B2, B5 and B8. In Sects. 7 and 8 we study respectively the effect of the geometrical thickness of the disk and the inclination angle between the line of sight and the rotational axis. Section 9 deals with the rotational and the terminal wind velocity fields and their influence on the $\operatorname{Br} \gamma$ line profile. Finally, in Sect. 10 we summarize and discuss our main results.

\section{SIMECA: A code dedicated to active hot stars}

The SIMECA code has been already described in previous papers (see Stee \& Araùjo 1994 and Paper I). It computes classical observables, i.e. spectroscopic and photometric ones but also intensity maps in Balmer lines and in the continuum in order to obtain theoretical visibility curves which can be directly compared to high angular resolution data. The main hypothesis of this code is that the envelope is axi-symmetric with respect to the rotational axis. No meridian circulation is allowed. We assume that the physics of the polar regions is well represented by a CAK type stellar wind model (Castor et al. 1975) and the solutions for all stellar latitudes are obtained by introducing a parametrized model which is constrained by spectrally resolved interferometric data. The inner equatorial region is dominated by Keplerian rotation.

In order to take into account the 7-4 levels radiative transition to reproduce the $\operatorname{Br} \gamma$ line profile at $2.16 \mu \mathrm{m}$, we have added one more level to the hydrogen atoms which have now seven bound levels. The ionizationexcitation equations are solved for an envelope modeled in a $170 * 90 * 71$ cube. Since the final population of atomic levels are strongly NLTE distributed we start with the LTE populations for each level, we then compute the escape probability of each transition which allows us to obtain up-dated populations, and we iterate until convergence. The convergence is quite fast (about ten iterations) and stable within an effective temperature of the central star in the range $10000<T_{\text {eff }}<40000$. The basic equations of the SIMECA code are given in detail in Paper I.

To account for the photospheric absorption line, we assumed the underlying star to be a normal B star with a given $T_{\text {eff }}$ and $\log g$. For each $T_{\text {eff }}$ and $\log g$ we have computed $\mathrm{H} \alpha, \mathrm{H} \beta$ and $\mathrm{Br} \gamma$ synthetic line profiles using the SYNSPEC code developed by Hubeny (Hubeny 1988; Hubeny \& Lanz 1995). These photospheric line profiles are then broadened by solid rotation and can be further absorbed by the envelope volume projected on the stellar disk. The SIMECA code is also able to produce theoretical intensity maps of the circumstellar envelope in these lines and in the continuum at different wavelengths (see for instance Fig. 1). These maps can be directly compared to milli-arcsecond interferometric measurements such as those obtained from the GI2T (Paper I) and Mark III (Quirrenbach et al. 1993) interferometers or with the forthcoming VLT interferometer.
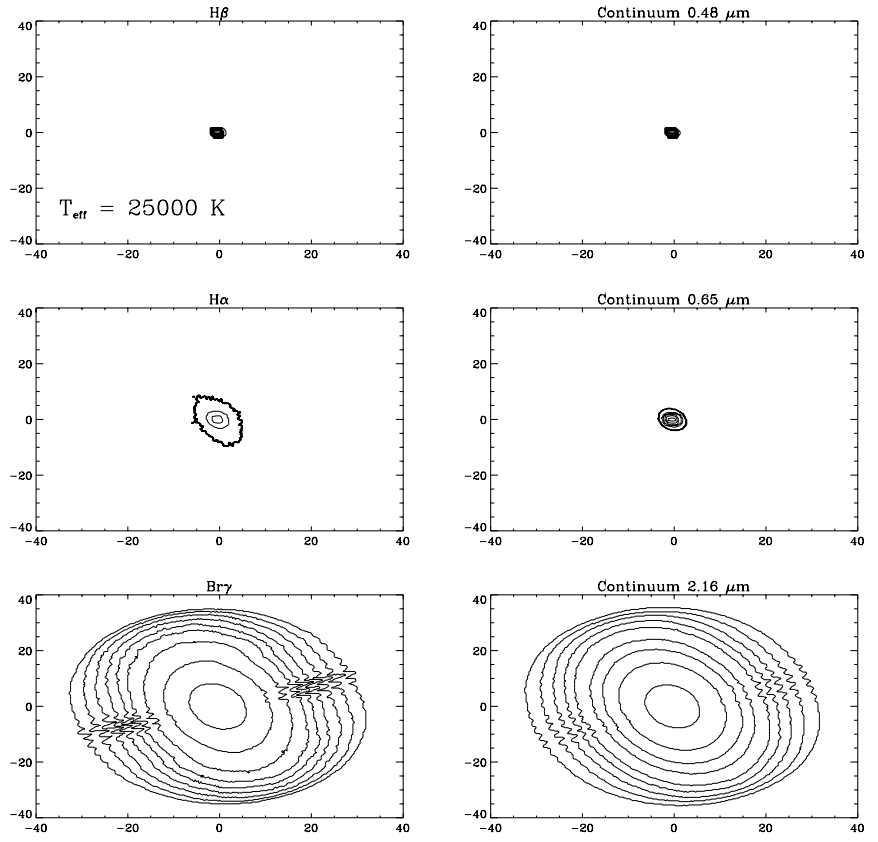

Fig. 1. Intensity maps with parameters representative of the Be Star $\gamma$ Cas (see Paper I and Table 1) computed with SIMECA. From the upper left to the lower right respectively in the $\mathrm{H} \beta$ line, Continuum at $0.48 \mu \mathrm{m}, \mathrm{H} \alpha$ line, Continuum at $0.65 \mu \mathrm{m}, \mathrm{Br} \gamma$ line, Continuum at $2.16 \mu \mathrm{m}$ within a spectral bandwith of $40 \AA$. The axes are in stellar radius. North is up, East is left

\section{The particular case of $\gamma$ Cas}

Among Be stars, $\gamma$ Cas (B0.5 IVe, HD 5394, HR 264) is certainly the most studied Be star. Its geometry has been already studied in detail by long baseline interferometry at visible wavelengths (Thom et al. 1986; Quirrenbach et al. 1993; Paper I; Quirrenbach et al. 1997). In the near IR, many studies have used spectroscopic data to constrain the physics of $\gamma$ Cas (Hamman \& Simmon 1987; Hony et al. 2000) but no observations aimed at a direct study of its circumstellar environment have been carried out yet. Using SIMECA, we have computed intensity maps in the $\operatorname{Br} \gamma$ line profile and in the continuum at $2.16 \mu \mathrm{m}$ (see Fig. 1) with stellar and wind parameters given in Table 1. Since we have added one more level to the hydrogen atoms in order to reproduce the $\mathrm{Br} \gamma$ line and since it changed the ionization-exitation equations, we have computed $\mathrm{H} \alpha$ and $\mathrm{H} \beta$ line profiles and intensity maps in order to check if it affected our previous results. From Fig. 5 it is clear that we obtain similar profiles and maps for $\mathrm{H} \alpha$ and $\mathrm{H} \beta$ compared to those obtained in Stee \& Araùjo (1994) and in Paper I. From the maps in Fig. 1 (plotted with the same iso-contour dynamic) it is clear that the extension of $\gamma$ Cas is very large in the near-IR. The maximal extention in the North-South direction is 41 stellar radii at $2.16 \mu \mathrm{m}$. This is mainly due to the fact that at IR wavelengths, the contribution from the star is less important compared to contribution from the envelope. Thus, the contrast between the star and the envelope is less pronounced and the objet (star+envelope) appears more extended. In other words, 
Table 1. Parameters and results obtained for $\gamma$ Cas with SIMECA (see Paper I for details)

\begin{tabular}{|c||c|}
\hline Spectral type & B0.5 IVe \\
\hline$T_{\text {eff }}$ & $25000 \mathrm{~K}$ \\
\hline Mass & $16 M_{\odot}$ \\
\hline Radius & $10 R_{\odot}$ \\
\hline Luminosity & $3.510^{4} L_{\odot}$ \\
\hline$V \cdot \sin i$ & $230 \mathrm{~km} \mathrm{~s}^{-1}$ \\
\hline Inclination angle $i$ & $45^{\circ}$ \\
\hline Photospheric density & $5.010^{-12} \mathrm{~g} \mathrm{~cm}^{-3}$ \\
\hline Photospheric expansion velocity & $0.11 \mathrm{~km} \mathrm{~s}^{-1}$ \\
\hline Equatorial rotation velocity & $326 \mathrm{~km} \mathrm{~s}^{-1}$ \\
\hline Equatorial terminal velocity & $200 \mathrm{~km} \mathrm{~s}^{-1}$ \\
\hline Polar terminal velocity & $2000 \mathrm{~km} \mathrm{~s}^{-1}$ \\
\hline Polar mass flux & $1.710^{-9} M_{\odot} \mathrm{year}^{-1} \mathrm{sr}^{-1}$ \\
\hline$m 1$ & 6.0 \\
\hline Mass of the disk & $4.4310^{-9} M_{\odot}$ \\
\hline Mass loss & $3.1610^{-7} M_{\odot} \mathrm{year}^{-1}$ \\
\hline
\end{tabular}

in the near-IR, the central star does not overwhelm the emission and it thus becomes possible to "see" the emission from the surrounding matter located at larger distances. To measure an angular extension, we compute the region where more than $50 \%$ of the total flux originates, then follow the gradient of the computed image intensity as a function of the stellar radius. If the gradient becomes smaller, implying that we are in the "halo" of the emitting region and not in the core, we return, stopping where the gradient is steeper in order to obtain a "clean" edge. This point corresponds to the angular extension given in Table 2. Since the modulus of the complex degree of coherence of an astrophysical source is equal to the modulus of the normalized spatial Fourier transform of the source's brightness, we have computed the modulus of the Fourier transform of these maps. Finally, in order to obtain theoretical visibilities, we have cut these $2 \mathrm{D}$ maps in the direction which corresponds to the GI2T baseline orientation (North-South). It can be seen in Fig. 2 that with baselines on the order of 50 meters the envelope is well resolved, even in the near-IR where the spatial resolution is lower by a factor of 4 , due to the longer wavelengths, compared to the visible (if $\mathrm{B}$ is the baseline and $\lambda$ is the wavelength the spatial resolution changes as $\lambda / \mathrm{B}$ ). In the continuum (Fig. 3) the differences are even larger: $\gamma$ Cas appears as a point source at $0.48 \mu \mathrm{m}$ and is poorly resolved at $0.65 \mu \mathrm{m}$, whereas at $2.16 \mu \mathrm{m}$ the extended free-free and free-bound envelope emission are expected to be very well resolved.

The difference between the calculated visibilities in the $\operatorname{Br} \gamma$ and the $2.16 \mu \mathrm{m}$ continuum is not very large since the $\operatorname{Br} \gamma$ line originates from the same region as its nearby continuum and the line emission is only $9 \%$ of the total (star+envelope) flux (see Col. 3 in Table 3 for $\left.\rho=510^{-12} \mathrm{~g} \mathrm{~cm}^{-3}\right)$. In Fig. 4 we have plotted the
Table 2. Envelope size of $\gamma$ Cas in units of stellar radii and milliarcsecond (mas) obtained with the SIMECA code (Wavelengths in $\mu \mathrm{m}$ are emission in the continuum)

\begin{tabular}{|c|c|c|}
\hline Wavelength & $\begin{array}{c}\text { Angular diameter } \\
\text { (stellar radii) }\end{array}$ & $\begin{array}{c}\text { Angular diameter } \\
\text { (mas) }\end{array}$ \\
\hline $\mathrm{H} \alpha$ & 15.6 & 3.51 \\
\hline $\mathrm{H} \beta$ & 8.1 & 1.82 \\
\hline $\mathrm{Br} \gamma$ & 43.1 & 9.69 \\
\hline $\mathrm{He} \mathrm{I} \quad 6678$ & $\sim 4$ & $\sim 0.9$ \\
\hline $0.65 \mu \mathrm{m}$ & 3.7 & 0.83 \\
\hline $0.48 \mu \mathrm{m}$ & 2.8 & 0.63 \\
\hline $2.16 \mu \mathrm{m}$ & 41.2 & 9.27 \\
\hline
\end{tabular}

ratio of the calculated visibilities in the lines and in the corresponding continuum (i.e. referenced visibilities). The crosses are data in the $\mathrm{H} \alpha$ line obtained from the GI2T during the 1993 campain. The agreement with our model is better for larger baselines, except for the last data point and, as already mentioned in Paper I, it seems that our model envelope is larger than the observed envelope. Since the visibilities in the $\operatorname{Br} \gamma$ and the $2.16 \mu \mathrm{m}$ continuum were similar, the referenced visibility at this wavelength is close to one. Thus the result from the cross-correlation data reduction method, where we cross-correlate a wide spectral channel taken in the continuum with a channel centered on the line, must show a nearly unresolved object whereas with the autocorrelation method, $\gamma$ Cas will be well resolved both in the line and in the continuum. Of course, both methods finally give the same referenced visibility but from a $\mathrm{S} / \mathrm{N}$ point of view it may be easier to use the cross-correlation method (with well contrasted fringes) while the autocorrelation method deals with well resolved visibilities (and poorly contrasted fringes). This was not the case in previous studies since $\gamma \mathrm{Cas} \mathrm{H} \alpha$ and $\mathrm{H} \beta$ continua were poorly resolved and thus the theoritical visibilities referenced or unreferenced were similar. Nevertheless, we must point out that for "real" data it is crucial to use referenced visibilities since they are in principle independent of seeing and instrumental noise.

We obtain for $\gamma$ Cas the extensions summarized in Table 2. In Fig. 2 it is also important to note that with $10 \mathrm{~m}$ class telescopes working in the diffraction limited mode (adaptive optics reference, aperture masking reference) it may be possible to resolve the envelope around $\gamma$ Cas in $\mathrm{H} \alpha, \mathrm{H} \beta$ and $\mathrm{Br} \gamma$ lines (see Tuthill et al. 2000 for an example using the Keck telescope in the aperture masking mode). Moreover, these telescopes may be complementary to long baseline interferometers such as GI2T where it is not possible to use baselines less than 16 meters, beyond which the models predict the extended component to be completely resolved. In Fig. 5 we present normalized line profiles and the spectral energy distribution curve obtained with SIMECA. The line profiles are asymmetric with $V / R<1$, and the maximum intensity decreasing following $\mathrm{H} \alpha, \mathrm{H} \beta$ and $\mathrm{Br} \gamma$. We notice that the $\mathrm{Br} \gamma$ line profile presents a large FWHM of $390 \mathrm{~km} \mathrm{~s}^{-1}$ which strongly depends on the wind terminal velocity (see Sect. 9). 


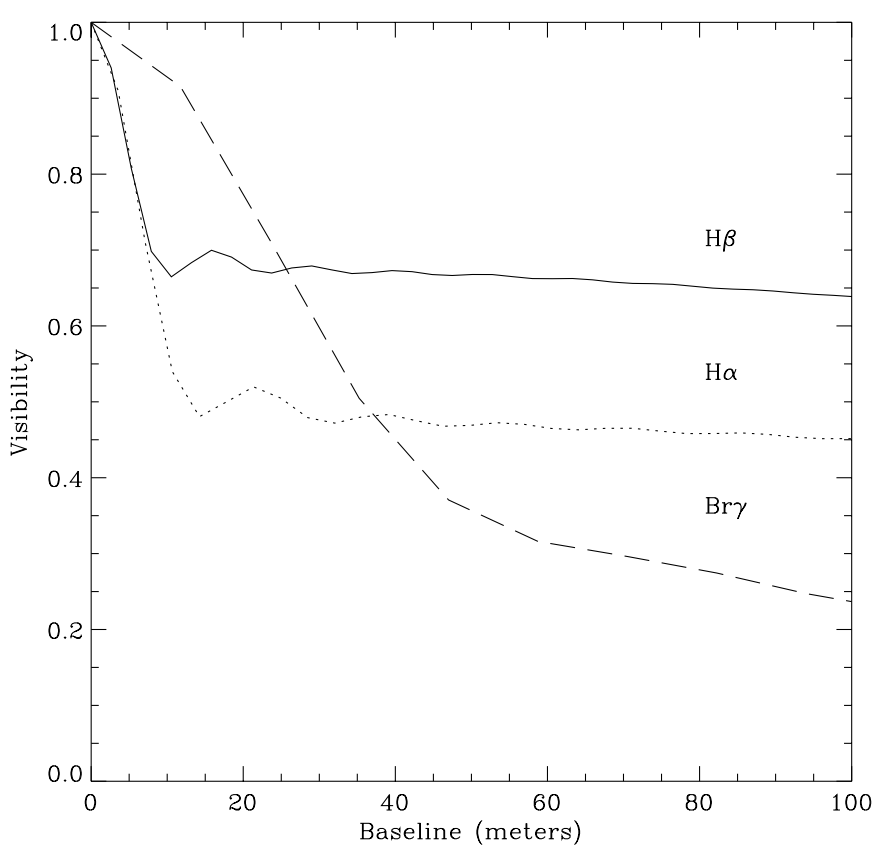

Fig. 2. Visibilities as a function of baseline (in meters) in the North-South direction for the Be Star $\gamma$ Cas. Respectively in the $\mathrm{H} \beta$ (solid line), $\mathrm{H} \alpha$ (dotted line) and $\mathrm{Br} \gamma$ (dashed line) line profiles

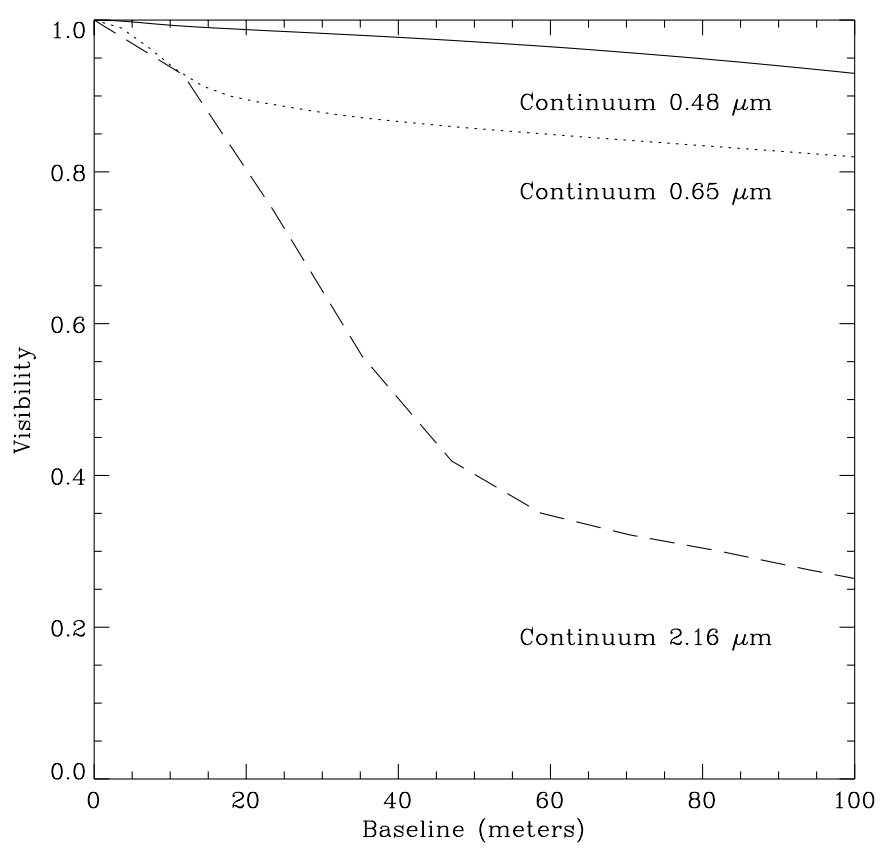

Fig. 3. Visibilities as a function of baseline (in meters) in the North-South direction for the Be Star $\gamma$ Cas. Respectively in the 0.48 (solid line), 0.65 (dotted line) and 2.16 (dashed line) $\mu \mathrm{m}$ continuum

The spectral energy distribution curve shows a large IR excess after $2 \mu \mathrm{m}$ due to the envelope free-free and freebound emission.

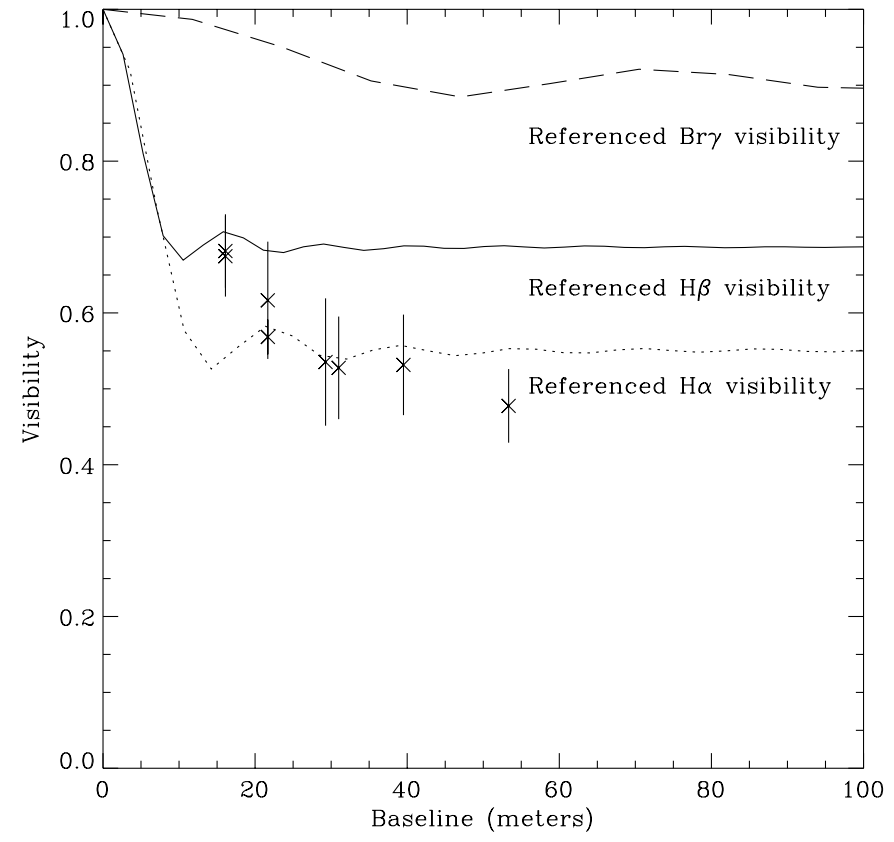

Fig. 4. Referenced visibilities as a function of baseline (in meters) in the North-South direction for the Be Star $\gamma$ Cas. Respectively in the $\mathrm{H} \beta$ (solid line), $\mathrm{H} \alpha$ (dotted line) and $\mathrm{Br} \gamma$ (dashed line) line profiles. The crosses are data in the $\mathrm{H} \alpha$ line obtained from the GI2T during the 1993 campaign (see Paper I for more details)

\section{Effect of the wind density}

We may wonder if these results for $\gamma$ Cas may be extended to other Be stars with different wind densities. The wind density range at the base of the photosphere, usually allowed for Be stars, lies between $10^{-14}$ and $10^{-11} \mathrm{~g} \mathrm{~cm}^{-3}$ (Gehrz et al. 1974; Dachs et al. 1988; Kastner \& Mazzali 1989; Hony et al. 2000) thus we have performed simulations within this range, keeping the other parameters constant and equal to those used for $\gamma$ Cas and defined in Table 1.

\subsection{Line profiles and spectral energy distribution}

From Fig. 6 it is clear that for very low densities (less than $510^{-14} \mathrm{~g} \mathrm{~cm}^{-3}$ ), the lines are not in emission and we only have a photospheric absorption line profile. For $\rho=510^{-11} \mathrm{~g} \mathrm{~cm}^{-3}$, the $\mathrm{H} \beta$ line shows the strongest emission. The $\mathrm{H} \alpha$ line profile also appears and shows very extended line wings, whereas the $\operatorname{Br} \gamma$ profile is just above the continuum level. This is due to the fact that for $\rho=510^{-11} \mathrm{~g} \mathrm{~cm}^{-3}$ there is a very large contribution from the envelope continuum (see Fig. 7 upper left) which can reach $50 \%$ and $98 \%$ of the total flux, respectively, for $\mathrm{H} \alpha$ and $\mathrm{Br} \gamma$, lowering the emission in the line (see Table 3). It is more obvious on the lower left of Fig. 7, where the envelope line emission increases in strength with increasing densities: up to $510^{-13} \mathrm{~g} \mathrm{~cm}^{-3}$ for $\operatorname{Br} \gamma$ and up to $510^{-12} \mathrm{~g} \mathrm{~cm}^{-3}$ for $\mathrm{H} \alpha$. For larger densities, the percentage of the line emission decreases except for $\mathrm{H} \beta$ 

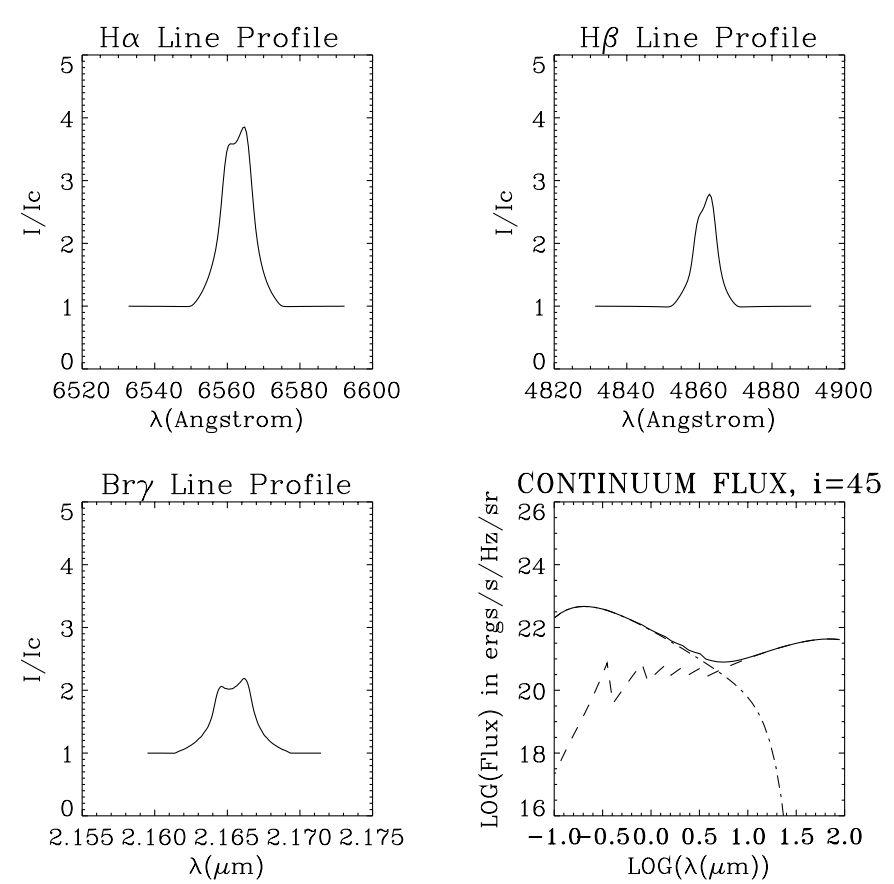

Fig. 5. Line profiles with parameters representative of the $\mathrm{Be}$ Star $\gamma$ Cas (see Paper I) computed with SIMECA. Lower right corner: energy distibution as a function of wavelength (dashed line: envelope flux, dash-dot line: stellar flux, solid line: total flux)

which increases from $510^{-14}$ to $510^{-10} \mathrm{~g} \mathrm{~cm}^{-3}$. There is a difference of 6 magnitudes between the $510^{-15}$ and $510^{-11} \mathrm{~g} \mathrm{~cm}^{-3}$ densities in the $\operatorname{Br} \gamma$ absolute fluxes (upper right in Fig. 7). This difference is only of 1.3 magnitudes for $\mathrm{H} \alpha$, whereas it remains nearly constant for $\mathrm{H} \beta$. The stellar emission (lower right in Fig. 7) is essentially the mirror of the envelope continuum emission, i.e. it decreases when the envelope continuum increases and vice versa.

In a paper by Hony et al. (2000), they used, as a model for the IR continuum energy distribution of $\gamma$ Cas, a stellar contribution to the total flux of about $20 \%$ at $2.4 \mu \mathrm{m}$, based on extrapolation of a Kurucz model atmosphere fitted to the UV continuum. We found for the same density, i.e. $510^{-11} \mathrm{~g} \mathrm{~cm}^{-3}$ that only $1 \%$ of the total flux originates from the central star. From our simulation, we obtain that the envelope density should be as low as $510^{-13} \mathrm{~g} \mathrm{~cm}^{-3}$ in order to obtain a similar (24\%) stellar contribution.

In the study by Zaal et al. (1995), they found that IR emission lines may be detectable for densities up to about $10^{-14} \mathrm{~g} \mathrm{~cm}^{-3}$. They also found that around this density, HI IR lines are in emission while Balmer lines do not show any emission because of the strong underlying continuum. For $\rho=510^{-13} \mathrm{~g} \mathrm{~cm}^{-3}$ we obtain the strongest IR line emission in $\operatorname{Br} \gamma(34.2 \%$ of the total flux) whereas for lower densities the emission is too faint to be detectable. For higher densities, due to an increase of the envelope underlying continuum (up to $98.4 \%$ for $\rho=510^{-11} \mathrm{~g} \mathrm{~cm}^{-3}$ ), the emission in the $\operatorname{Br} \gamma$ line will also decrease. Thus in order
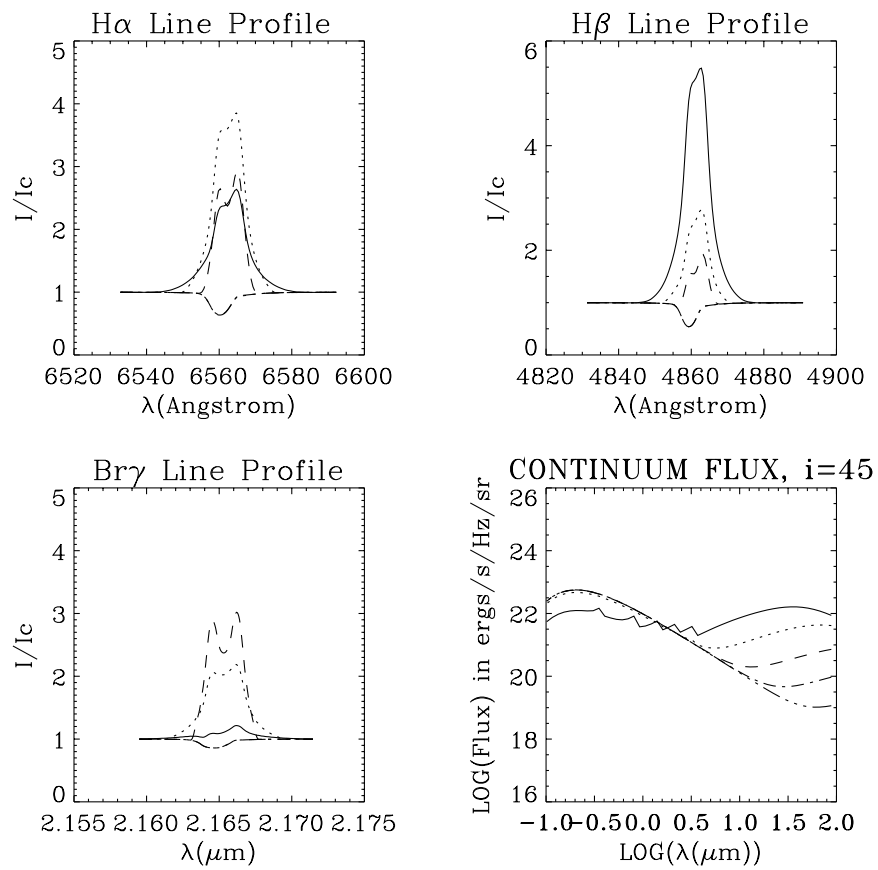

Fig. 6. Line profiles with different wind density at the base of the photosphere. Respectively: $\rho=510^{-11}$ (solid line), $510^{-12}$ (dotted), $510^{-13}$ (dashed), $510^{-14}$ (dash-dot) and $510^{-15} \mathrm{~g} \mathrm{~cm}^{-3}$ (dash-dot-dot-dot) computed with SIMECA. Lower right corner: energy distibution as a function of wavelength (with density plots convention as for the line)

to have the largest probability of HI IR lines in emission, the density must be close to $510^{-13} \mathrm{~g} \mathrm{~cm}^{-3}$ which is a factor of 100 lower than typical values for Be stars. We were not able to obtain HI IR lines in emission with Balmer lines in absorption with the same stellar parameters. The results of our simulations are summarized in Table 3.

\subsection{Visibilities}

We have also studied the effect of changing the density on the visibility curves. From Fig. 8 we can see that the visibilites are very sensitive to a density change. As for $\gamma$ Cas, the $\mathrm{Br} \gamma$ line and its nearby continuum are the most extended emitting regions. For very dense envelopes, the $\mathrm{Br} \gamma$ visibility falls down to 0.2 for a baseline of 100 meters as does the $\mathrm{H} \alpha$ visibility. For $\rho=510^{-14}$ and $510^{-15} \mathrm{~g} \mathrm{~cm}^{-3}$ the emitting regions are too faint to be resolved in the $\mathrm{H} \alpha$ and $\mathrm{H} \beta$ lines or in their nearby continua. In the $\mathrm{H} \beta$ continuum the density must be on the order of $510^{-11} \mathrm{~g} \mathrm{~cm}^{-3}$ in order to be barely resolved with baseline of a few meters whereas in the $\mathrm{H} \alpha$ continuum a density of $510^{-12} \mathrm{~g} \mathrm{~cm}^{-3}$ is sufficient. Measuring the visibilities in these 3 lines and their continua may be a simple way to deduce the density of the envelope of Be stars. 
Table 3. Relative fluxes as a function of the wind density at the base of the photosphere

\begin{tabular}{|c|c|c|c|c|}
\hline$T_{\text {eff }}=25000 \mathrm{~K}$ & $\begin{array}{c}\text { Stellar contribution } \\
(\text { in } \%) \\
\end{array}$ & $\begin{array}{c}\text { Envelope contribution } \\
\text { in the line (in \%) }\end{array}$ & $\begin{array}{c}\text { Envelope contribution } \\
\text { in the continuum (in \%) }\end{array}$ & $\begin{array}{c}\text { Total } \\
\left(\text { in } 10^{32} \mathrm{erg} \mathrm{s}^{-1}\right) \\
\end{array}$ \\
\hline \multicolumn{5}{|l|}{$\rho=510^{-11} \mathrm{~g} \mathrm{~cm}^{-3}$} \\
\hline $\mathrm{H} \alpha$ & 11.2 & 37.4 & 62.5 & 30 \\
\hline $\mathrm{H} \beta$ & 32.8 & 51.0 & 48.9 & 28 \\
\hline $\mathrm{Br} \gamma$ & 0.1 & 1.5 & 98.4 & 27 \\
\hline 0.65 & 17.9 & - & 82 & 19 \\
\hline 0.48 & 67.1 & - & 32.9 & 14 \\
\hline 2.16 & 0.09 & - & 99.91 & 27 \\
\hline \multicolumn{5}{|l|}{$\rho=510^{-12} \mathrm{~g} \mathrm{~cm}^{-3}$} \\
\hline $\mathrm{H} \alpha$ & 40.7 & 47.9 & 11.4 & 20 \\
\hline $\mathrm{H} \beta$ & 63.4 & 34.5 & 2.1 & 34 \\
\hline $\operatorname{Br} \gamma$ & 2.2 & 9.2 & 88.6 & 4.3 \\
\hline 0.65 & 78.2 & - & 21.8 & 10 \\
\hline 0.48 & 96.8 & - & 3.2 & 23 \\
\hline 2.16 & 2.4 & - & 97.6 & 3.9 \\
\hline \multicolumn{5}{|l|}{$\rho=510^{-13} \mathrm{~g} \mathrm{~cm}^{-3}$} \\
\hline $\mathrm{H} \alpha$ & 64.0 & 34.6 & 1.4 & 9.3 \\
\hline $\mathrm{H} \beta$ & 80.2 & 19.6 & 0.2 & 25 \\
\hline $\operatorname{Br} \gamma$ & 15.5 & 34.2 & 50.3 & 0.45 \\
\hline 0.65 & 97.9 & - & 2.1 & 9.3 \\
\hline 0.48 & 99.7 & - & 0.3 & 25 \\
\hline 2.16 & 23.6 & - & 76.4 & 0.45 \\
\hline \multicolumn{5}{|l|}{$\rho=510^{-14} \mathrm{~g} \mathrm{~cm}^{-3}$} \\
\hline $\mathrm{H} \alpha$ & 99.6 & 0.2 & 0.2 & 9.2 \\
\hline $\mathrm{H} \beta$ & 99.9 & 0 & 0.1 & 25 \\
\hline $\operatorname{Br} \gamma$ & 75.3 & 0.1 & 50.3 & 0.14 \\
\hline 0.65 & 99.8 & - & 0.2 & 9.2 \\
\hline 0.48 & 99.9 & - & 0.1 & 25 \\
\hline 2.16 & 75.3 & - & 24.7 & 0.14 \\
\hline \multicolumn{5}{|l|}{$\rho=510^{-15} \mathrm{~g} \mathrm{~cm}^{-3}$} \\
\hline $\mathrm{H} \alpha$ & 100 & 0 & 0 & 8.9 \\
\hline $\mathrm{H} \beta$ & 100 & 0 & 0 & 25 \\
\hline $\mathrm{Br} \gamma$ & 96.8 & 0 & 3.2 & 0.11 \\
\hline 0.65 & 99.9 & - & 0.02 & 9.2 \\
\hline 0.48 & 100 & - & 0 & 25 \\
\hline 2.16 & 96.8 & - & 3.2 & 0.11 \\
\hline
\end{tabular}

\section{Effect of the effective temperature}

We have investigated the effect of changing the effective temperature $T_{\text {eff }}$ of the central star. We recall that in SIMECA the envelope temperature follows:

$T(r) \propto \frac{T_{\text {eff }}}{r^{\frac{1}{2}}}$

where $r$ is the distance from the central star in unit of stellar radius.

\subsection{Line profiles and spectral energy distribution}

From Fig. 9 we see that with decreasing temperature the equivalent width of all the lines, or the line-to-continuum ratio, increases. This result has already been found by Marlborough et al. (1997) for the $\mathrm{H} \alpha$ and $\mathrm{Br} \alpha$ line profiles, although the temperature range for their study was between 2000 and $10000 \mathrm{~K}$ whereas we investigate a temperature range between 16000 and $40000 \mathrm{~K}$. They also found that the line width increases with increasing temperature, which is not the case in Fig. 9. The main reason is due to the fact that in Marlborough et al. (1997) they used 

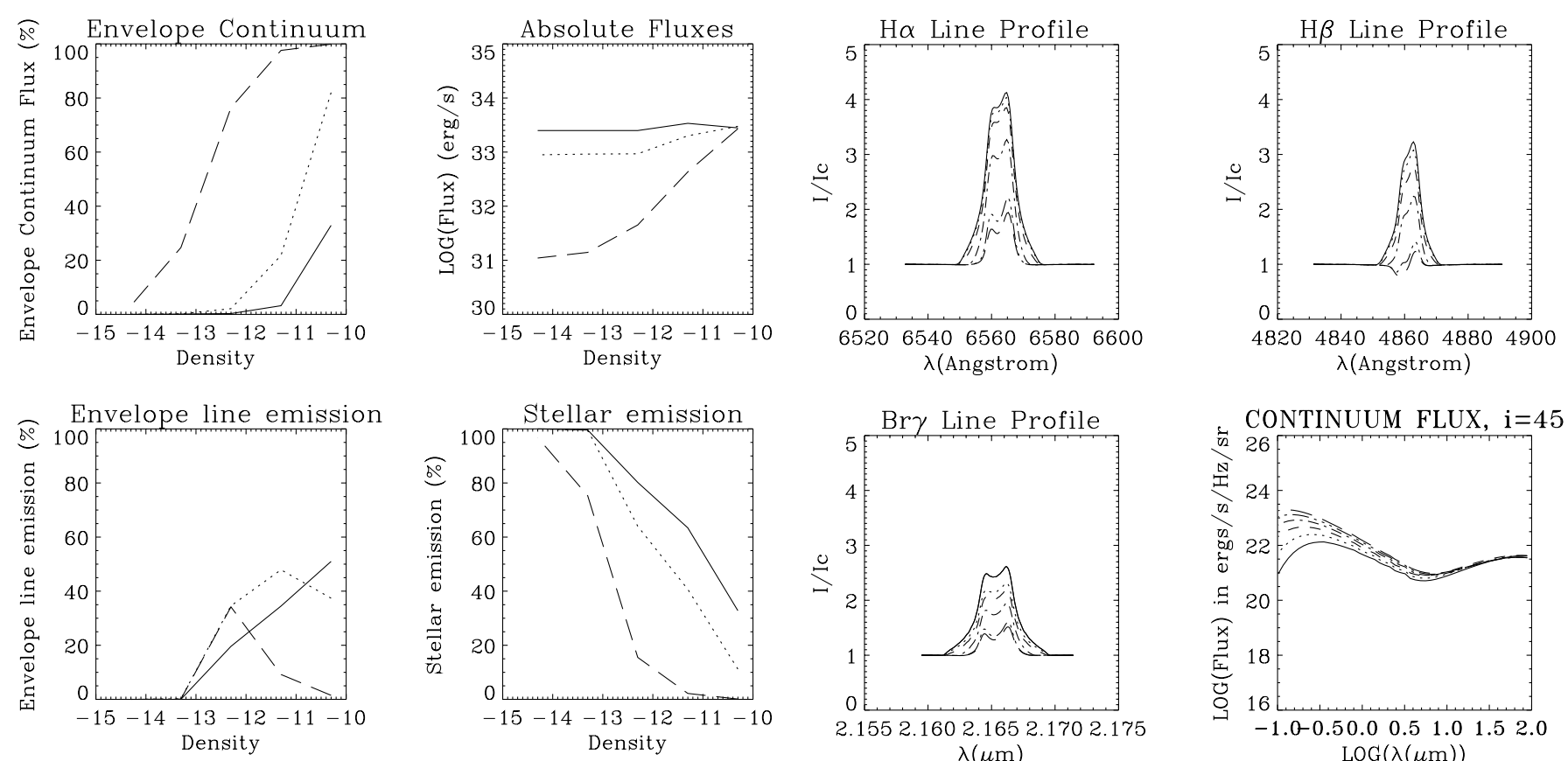

Fig. 7. Different flux contributions (in \%) as a function of the wind density at the base of the photosphere ( $\log$ scale). For all plots we have the following convention: $\mathrm{H} \beta$ or $0.48 \mu \mathrm{m}$ (solid line), $\mathrm{H} \alpha$ or $0.65 \mu \mathrm{m}$ (dotted line), $\operatorname{Br} \gamma$ or $2.16 \mu \mathrm{m}$ (dashed line). Upper left: envelope continuum flux, Upper right: Log of the absolute fluxes in erg/s. Lower left: emission of the envelope in the line. Lower right: stellar contribution
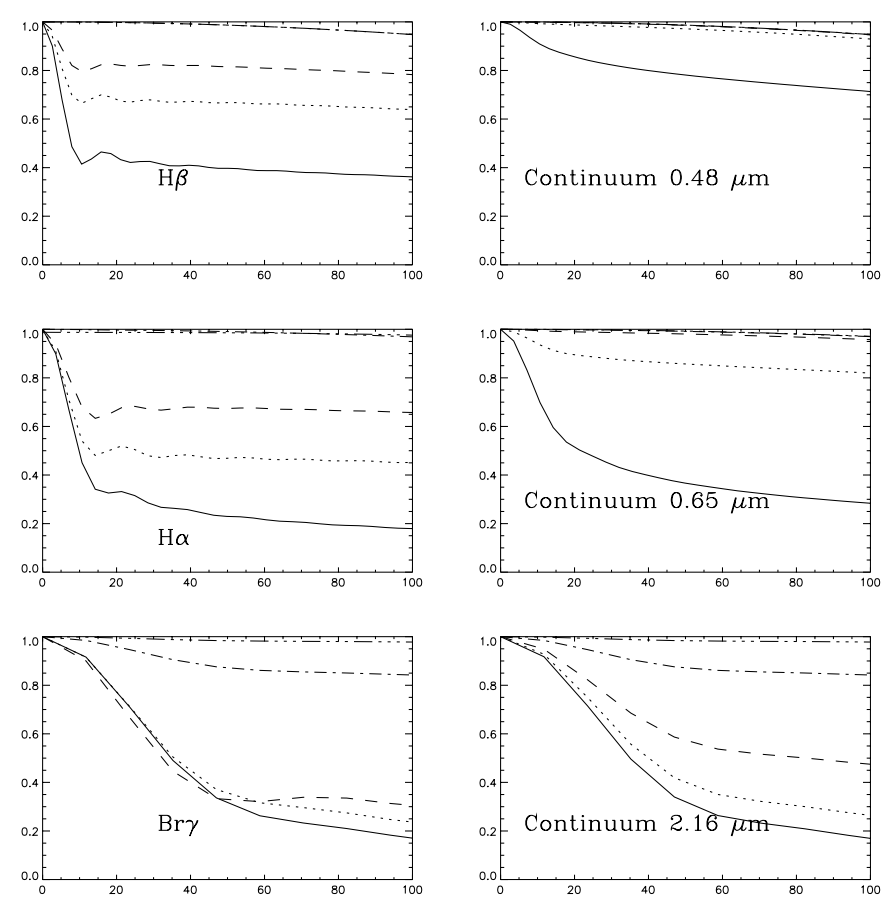

Fig. 8. Density effect on the visibility curves as a function of baseline (in meters) for different lines: $\mathrm{H} \beta$ and its nearby continuum (upper graph), $\mathrm{H} \alpha$ and its nearby continuum (middle graph) and $\operatorname{Br} \gamma$ and its continuum (lower graph). For each graph the curves are the following: $\rho=510^{-11}$ (solid line), $510^{-12}$ (dotted), $510^{-13}$ (dashed), $510^{-14}$ (dash-dot) and $510^{-15} \mathrm{~g} \mathrm{~cm}^{-3}$ (dash-dot-dot-dot)
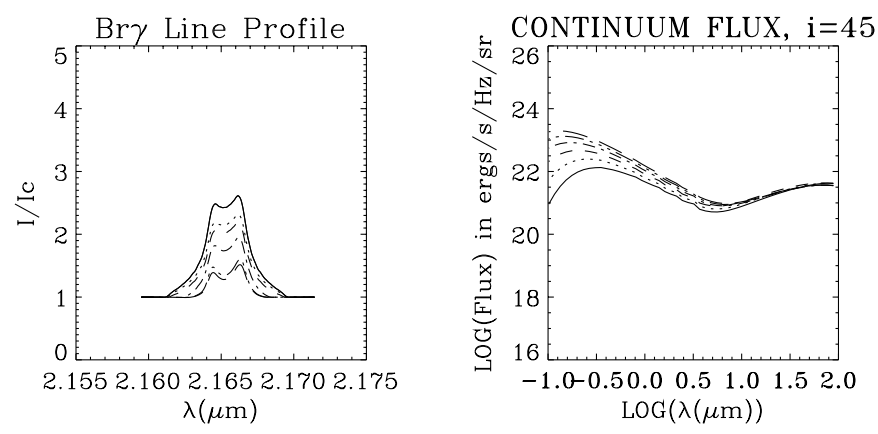

Fig. 9. Line profiles with different effective temperatures. Respectively: $T_{\text {eff }}=16000 \mathrm{~K}$ (solid line), $20000 \mathrm{~K}$ (dotted), 25000 (dashed), 30000 (dash-dot), 35000 (dash-dot-dotdot) and 40000 (long-dashed) computed with SIMECA. Lower right: energy distibution as a function of wavelength (with temperature plots as for the line)

a Planck function as the line source function which decreases with decreasing temperature. Since the disc is optically thick, the line strength in the line wings is lower for lower temperature and thus the line width decreases with decreasing temperature. In SIMECA we solved the coupled ionization-excitation equations and our final atomic levels are strongly NLTE-distributed, as found by Hony et al. (2000) from the study of the infrared spectrum of $\gamma$ Cas. For 35000 and $40000 \mathrm{~K}$ the $\mathrm{H} \beta$ line presents a P-Cygni profile which can be due to the fact that the envelope continuum emission is strongly increasing with increasing temperature in the blue part of the spectrum. Thus the emission in the $\mathrm{H} \beta$ line is reduced (see lower right in Fig. 9). The $\operatorname{Br} \gamma$ line presents very extended wings up to $30 \AA$.

\subsection{Visibilities}

As for the study on the density effect, we have computed visibilities as a function of baseline for the same temperature range, keeping the other parameters constant. From Fig. 10 it is clear that the visibility curves are not very sensitive to a temperature change, compared to a density change especially for the continuum and the $\mathrm{Br} \gamma$ curves. Nevertheless, within the given temperature range the $\mathrm{Br} \gamma$-emitting region remains the most extended region. For all the lines, the visibility curves in the continuum are not strongly affected by temperature. Since the continuum originates mainly from free-free and freebound transitions and since the envelope is completely 

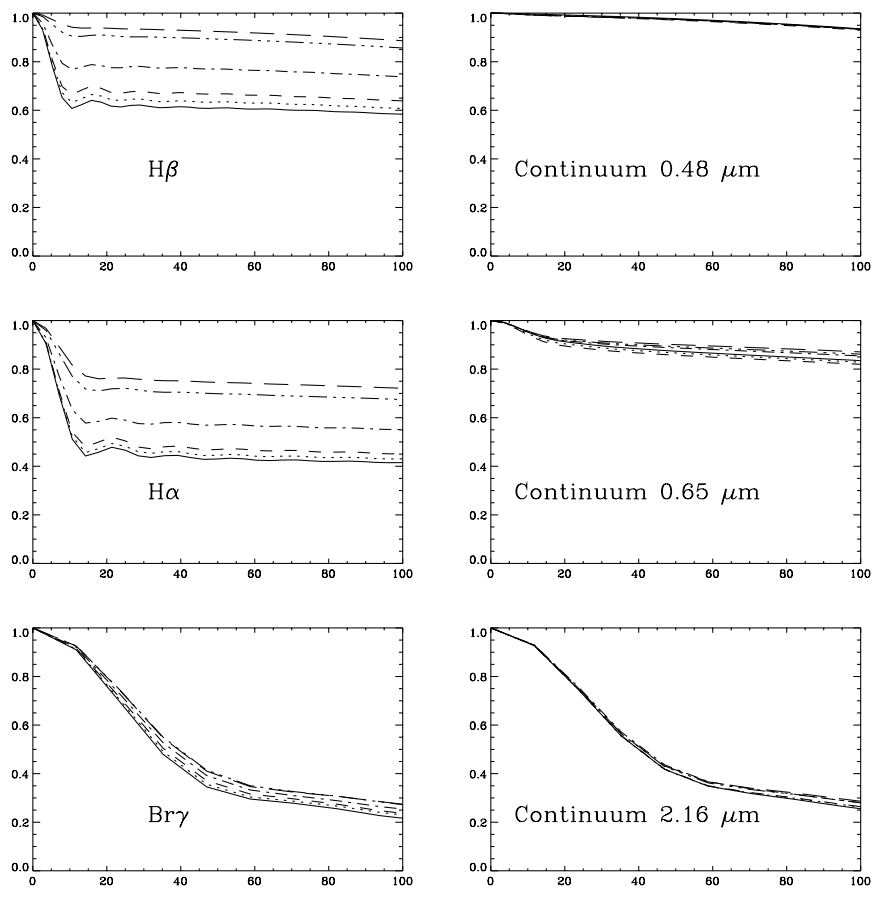

Fig. 10. Temperature effect on the visibility curves as a function of baseline (in meters) for different lines: $\mathrm{H} \beta$ and its nearby continuum (upper graph), $\mathrm{H} \alpha$ and its nearby continuum (middle graph) and $\mathrm{Br} \gamma$ and its continuum (lower graph). For each graph the curves are the following: $16000 \mathrm{~K}$ (solid line), $20000 \mathrm{~K}$ (dotted), $25000 \mathrm{~K}$ (dashed), $30000 \mathrm{~K}$ (dash-dot), $35000 \mathrm{~K}$ (dash-dot-dot-dot) and $40000 \mathrm{~K}$ (long-dashed)

ionized between 16000 and $40000 \mathrm{~K}$, it is not surprising that the continuum emitting regions are not very sensitive to the temperature. For the emitting regions in the lines, we found that the extensions decrease, i.e. the visibilities are closer to one, for increasing temperatures. This last result follows the decrease of the line-to-continuum ratio with increasing temperature, already outlined in the previous subsection.

\subsection{Correlation $L_{H \alpha}-L_{I R}$ with temperature}

Following the study by Neto \& de Freitas Pacheco (1982) and Kastner \& Mazzali (1989) who found an empirical relationship $\left(L_{\mathrm{H} \alpha}-L_{\mathrm{IR}}\right)$ between the luminosity in $\mathrm{H} \alpha$ and in the near-IR which allowed them to derive the central densities of Be discs, i.e. from $210^{11} \mathrm{~cm}^{-3}$ for late subtype to $310^{12} \mathrm{~cm}^{-3}$ for early sub-type, as well as the mass of the discs. We have studied whether such a correlation could exist for a given density in the disc $\left(310^{12} \mathrm{~cm}^{-3}\right)$ but for different effective temperatures.

Figure 11 indicates that there is such a correlation, approximately given by:

$\log \left(L_{\mathrm{H} \alpha}\right)=2.05 \log \left(L_{\mathrm{IR}}\right)-33.58$.

Note that the luminosities were determined using a spectral bandwith of $40 \AA$ for $\mathrm{H} \alpha$ and the IR. Thus in order to use Eq. (2) for comparison with other bandwiths or data

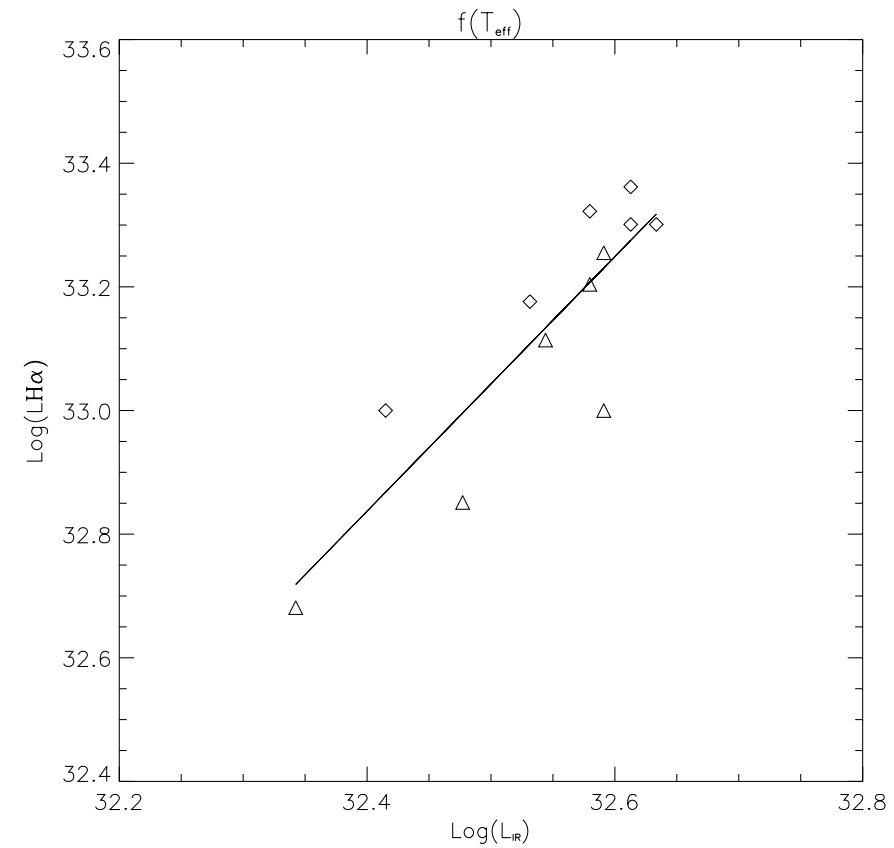

Fig. 11. Correlation $L_{\mathrm{H} \alpha}-L_{\mathrm{IR}}$ with temperature. Diamonds indicate luminosity determination from the line $(\mathrm{H} \alpha$ and $\mathrm{Br} \gamma$ for the IR emission) whereas triangles indicate determination from the continua at $0.65 \mu \mathrm{m}$ and at $2.16 \mu \mathrm{m}$. All fluxes are taken within a spectral bandwidth of $40 \AA$. Temperature increases from the lower left to the upper right (within $16000-$ $40000 \mathrm{~K})$. Units are in $\log \left(\mathrm{erg} \mathrm{s}^{-1}\right)$

we have to convolve the theoretical fluxes with the filter profiles to obtain the corresponding photometric fluxes. We will see in the following sections that this correlation is even more evident when we plot the same graph as a function of the B spectral type.

\subsection{Percentage of emission in the lines and in the continuum}

We have investigated how the fraction of envelope contribution to the total radiation in the lines and in the continuum is affected by temperature change. In Fig. 12 (upper left), we can see that the envelope continuum contribution, for the whole temperature range, is:

- in $\mathrm{H} \beta$ only a few (2-3\%) percent;

- in $\mathrm{H} \alpha$ about $20 \%$ for $15000<T_{\text {eff }}<26000 \mathrm{~K}$ and $15 \%$ for higher temperature;

- about $95 \%$ for $\operatorname{Br} \gamma$.

For the $\mathrm{H} \alpha$ and $\mathrm{H} \beta$ lines, the envelope contibution decreases with increasing temperature (lower left in Fig. 12), slowly between 15000 and $26000 \mathrm{~K}$ and more rapidly between 26000 and $35000 \mathrm{~K}$ whereas it becomes flatter after $35000 \mathrm{~K}$. For the $\mathrm{Br} \gamma$ line, the envelope emission slowly decreases but remains around $10 \%$ in the given temperature range. The stellar emission (lower right in Fig. 12) is very low for $\mathrm{Br} \gamma$ and increases with temperature for $\mathrm{H} \alpha$ and $\mathrm{H} \beta$. The absolute fluxes (upper right in Fig. 12) are not very different for $\mathrm{H} \alpha$ and $\mathrm{H} \beta$, whereas the absolute 

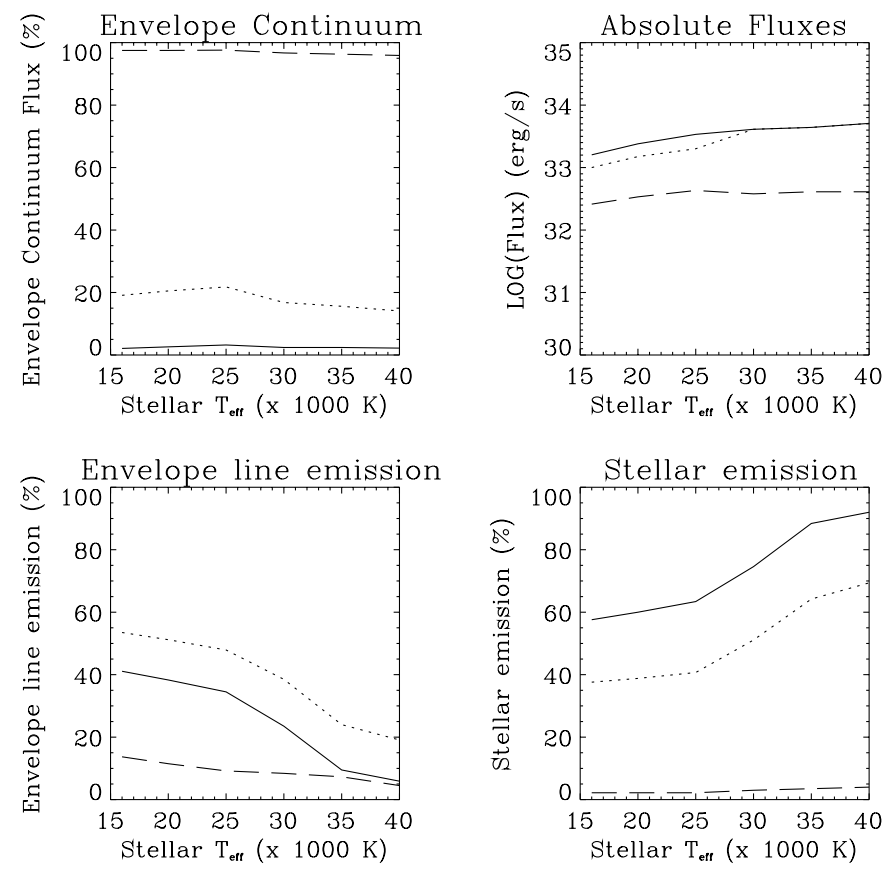

Fig. 12. Different flux contributions (in \%) as a function of the effective temperature (in unit of $1000 \mathrm{~K}$ ). For all plots we have the following convention: $\mathrm{H} \beta$ or $0.46 \mu \mathrm{m}$ (solid line), $\mathrm{H} \alpha$ or $0.65 \mu \mathrm{m}$ (dotted line), $\operatorname{Br} \gamma$ or $2.16 \mu \mathrm{m}$ (dashed line). Upper left: envelope continuum flux, Upper right: Log of the absolute fluxes in erg $\mathrm{s}^{-1}$. Lower left: emission of the envelope in the line. Lower right: stellar contribution

flux is a factor of 10 lower in $\mathrm{Br} \gamma$. We recall that the absolute fluxes were determined using a spectral bandwith of $40 \AA$ for $\mathrm{H} \alpha, \mathrm{H} \beta$ and the IR contributions. The results for the different models are summarized in Table 4.

\section{Effect of the spectral type}

In the previous section, we studied the effect of changing the effective temperature of the central star. In order to study the effect of changing the spectral type on our results we have computed models for different $\mathrm{B}$ spectral types: B0, B2, B5 and B8. We have adopted the same stellar parameters (see Table 5) given in Zaal et al. (1995) and have assumed for all the models that the density at the base of the photosphere is constant and equal to $510^{-12} \mathrm{~g} \mathrm{~cm}^{-3}$. From early to late spectral type the temperature and the radius both decrease. Thus, the relative fluxes in the lines and in the continuum are very different from those in Fig. 12 where temperature was the only free parameter. From Fig. 13 (upper right) we can see that the absolute fluxes (in erg $\mathrm{s}^{-1}$ ) decrease from early to late spectral type with a loss of 5 magnitudes for $\mathrm{Br} \gamma$ and 3 magnitudes for $\mathrm{H} \alpha$ and $\mathrm{H} \beta$. The stellar emission in Fig. 13 (lower right) does not increase for hotter stars as in Fig. 12 but is more or less constant as a function of the spectral type. The behaviour of the envelope line emission (lower left in Fig. 13) is also nearly constant for $\mathrm{H} \alpha$ and $\mathrm{H} \beta$ and increases from early to late spectral type for $\operatorname{Br} \gamma$. The envelope continuum emission is about $1.5 \%$ at $0.46 \mu \mathrm{m}$, about $10 \%$ at $0.65 \mu \mathrm{m}$ and $95 \%$ at $2.16 \mu \mathrm{m}$ and seems to be more or less independent of the spectral type (see Fig. 13, upper left).

In the paper by Zaal et al. (1995), they found that normal early B type stars have the strongest probability of being surrounded by a low-density disk which can be detected thanks to the HI IR lines in emission. Our results for a density of $510^{-12} \mathrm{~g} \mathrm{~cm}^{-3}$, illustrated in Table 6 , show the reverse: the envelope contribution in the $\mathrm{Br} \gamma$ line increases for later B-type stars, mainly due to a decrease of the envelope continuum contribution. Thus, for a given density, the probability of detecting HI IR lines in emission should be stronger for normal late B-type stars. This may explain why $70 \%$ of the 66 isolated Be stars within spectral types 09-B9 obtained by Clark \& Steele (2000) show Br $\gamma$ in emission. Within these $70 \%, 60 \%$ are between spectral types B0-B4 and thus $40 \%$ between B5-B9. Nevertheless, this result strongly depends on the assumed wind density.

Since surveys in the Paschen lines seem to indicate that no IR lines are detected for late B spectral types (Hubert, private communication) it may be an indication that:

- the density in the disc is very low $\left(\sim 10^{-14} \mathrm{~g} \mathrm{~cm}^{-3}\right)$ and near-IR emission lines are not detected but in this case the density is also too low to produce Balmer lines in emission;

- the density in the disc is very high $\left(\sim 10^{-11} \mathrm{~g} \mathrm{~cm}^{-3}\right)$ and due to the strong underlying continuum from the envelope, the line to continuum ratio is smaller in the near-IR lines, whereas Balmer lines are still visible.

From Table 3 it seems that around a density of $510^{-13} \mathrm{~g} \mathrm{~cm}^{-3}$ it is possible to have an important envelope contribution in the $\mathrm{Br} \gamma$ line and a similar or even smaller emission in the Balmer lines. This result agrees well with the density of $210^{-13} \mathrm{~g} \mathrm{~cm}^{-3}$ found by Zaal et al. (1995) from a theoretical growth curve applied to the B0.2V star $\tau$ Sco. This density was needed in order to obtain strong IR HI emission lines without noticeable emission in the photospheric $\mathrm{H} \alpha$ absorption line. All our results for different spectral types are summarized in Table 6 .

\subsection{Correlation $L_{H \alpha}-L_{I R}$ with the spectral type}

As for the temperature, we have studied if it is possible to determine an empirical relationship $\left(L_{\mathrm{H} \alpha}-L_{\mathrm{IR}}\right)$ between the luminosity in $\mathrm{H} \alpha$ and in the infrared as a function of the spectral type. From Fig. 14 we can see that the correlation is very good. We obtain the relation:

$\log \left(L_{\mathrm{H} \alpha}\right)=0.87 \log \left(L_{\mathrm{IR}}\right)+4.92$.

We have compared this relation with the Be star data obtained by Kastner \& Mazzali (1989) for a sample of 21 Be stars. In Fig. 15 we can see that the slope of the full line taken from Eq. (3) is in better agreement compared to the dashed line obtained from Eq. (2). In order to obtain a better agreement with the data points we were obliged 
Table 4. Relative fluxes as a function of the effective temperature

\begin{tabular}{|c|c|c|c|c|}
\hline$\rho=510^{-12} \mathrm{~g} \mathrm{~cm}^{-3}$ & $\begin{array}{c}\text { Stellar contribution } \\
\text { (in \%) } \\
\end{array}$ & $\begin{array}{l}\text { Envelope contribution } \\
\text { in the line (in \%) }\end{array}$ & $\begin{array}{c}\text { Envelope contribution } \\
\text { in the continuum (in \%) }\end{array}$ & $\begin{array}{c}\text { Total } \\
\left(\text { in } 10^{32} \mathrm{erg} \mathrm{s}^{-1}\right) \\
\end{array}$ \\
\hline \multicolumn{5}{|l|}{$\overline{T_{\text {eff }}=16000 \mathrm{~K}}$} \\
\hline $\mathrm{H} \alpha$ & 37.6 & 53.5 & 8.9 & 10 \\
\hline $\mathrm{H} \beta$ & 57.6 & 41.1 & 1.3 & 16 \\
\hline $\mathrm{Br} \gamma$ & 2.2 & 13.7 & 84.1 & 2.6 \\
\hline $0.65 \mu \mathrm{m}$ & 80.9 & - & 19.1 & 4.8 \\
\hline $0.48 \mu \mathrm{m}$ & 97.9 & - & 2.1 & 9.4 \\
\hline $2.16 \mu \mathrm{m}$ & 2.5 & - & 97.5 & 2.2 \\
\hline \multicolumn{5}{|l|}{$T_{\text {eff }}=20000 \mathrm{~K}$} \\
\hline $\mathrm{H} \alpha$ & 38.8 & 51.2 & 10.0 & 15 \\
\hline$\overline{\mathrm{H} \beta}$ & 60.0 & 38.3 & 1.7 & 24 \\
\hline $\mathrm{Br} \gamma$ & 2.2 & 8.4 & 88.6 & 3.4 \\
\hline $0.65 \mu \mathrm{m}$ & 79.5 & - & 16.8 & 7.1 \\
\hline $0.48 \mu \mathrm{m}$ & 97.4 & - & 2.6 & 15 \\
\hline $2.16 \mu \mathrm{m}$ & 2.5 & - & 96.7 & 3 \\
\hline \multicolumn{5}{|l|}{$T_{\text {eff }}=25000 \mathrm{~K}$} \\
\hline $\mathrm{H} \alpha$ & 40.7 & 47.9 & 11.4 & 20 \\
\hline $\mathrm{H} \beta$ & 63.4 & 34.5 & 2.1 & 34 \\
\hline $\operatorname{Br} \gamma$ & 2.2 & 9.2 & 88.6 & 4.3 \\
\hline $0.65 \mu \mathrm{m}$ & 78.2 & - & 21.8 & 10 \\
\hline $0.48 \mu \mathrm{m}$ & 96.8 & - & 3.2 & 23 \\
\hline $2.16 \mu \mathrm{m}$ & 2.4 & - & 97.6 & 3.9 \\
\hline \multicolumn{5}{|l|}{$T_{\text {eff }}=30000 \mathrm{~K}$} \\
\hline $\mathrm{H} \alpha$ & 51.1 & 38.5 & 10.4 & 21 \\
\hline $\mathrm{H} \beta$ & 74.6 & 23.5 & 1.9 & 41 \\
\hline $\mathrm{Br} \gamma$ & 3 & 8.4 & 88.6 & 3.8 \\
\hline $0.65 \mu \mathrm{m}$ & 83.2 & - & 16.8 & 13 \\
\hline $0.48 \mu \mathrm{m}$ & 97.6 & - & 2.4 & 31 \\
\hline $2.16 \mu \mathrm{m}$ & 3.3 & - & 96.7 & 3.5 \\
\hline \multicolumn{5}{|l|}{$T_{\text {eff }}=35000 \mathrm{~K}$} \\
\hline $\mathrm{H} \alpha$ & 64.2 & 24 & 11.8 & 20 \\
\hline $\mathrm{H} \beta$ & 88.4 & 9.5 & 2.1 & 44 \\
\hline $\operatorname{Br} \gamma$ & 3.5 & 7.3 & 89.2 & 4.1 \\
\hline $0.65 \mu \mathrm{m}$ & 84.4 & - & 15.6 & 16 \\
\hline $0.48 \mu \mathrm{m}$ & 97.6 & - & 2.4 & 39 \\
\hline $2.16 \mu \mathrm{m}$ & 3.7 & - & 96.3 & 3.8 \\
\hline \multicolumn{5}{|l|}{$T_{\text {eff }}=40000 \mathrm{~K}$} \\
\hline $\mathrm{H} \alpha$ & 69.4 & 19.1 & 11.5 & 23 \\
\hline $\mathrm{H} \beta$ & 92.0 & 5.9 & 2.1 & 51 \\
\hline $\mathrm{Br} \gamma$ & 4.0 & 4.5 & 91.5 & 4.1 \\
\hline $0.65 \mu \mathrm{m}$ & 85.9 & - & 14.1 & 18 \\
\hline $0.48 \mu \mathrm{m}$ & 97.8 & - & 2.2 & 48 \\
\hline $2.16 \mu \mathrm{m}$ & 4.1 & - & 95.9 & 3.9 \\
\hline
\end{tabular}

to translate the full line by 1.46 along the $\log \left(L_{\mathrm{H} \alpha}\right)$ axis, i.e. the full line in Fig. 15 now follows:

$\log \left(L_{\mathrm{H} \alpha}\right)=0.87 \log \left(L_{\mathrm{IR}}\right)+3.46$.

This translation reflects the fact that using Eq. (3) we obtain $\mathrm{H} \alpha$ luminosities which are too high compared to the data of Kastner \& Mazzali. Nevertheless, we recall that the $\mathrm{H} \alpha$ luminosities in Kastner \& Mazzali were obtained using the mean $\mathrm{H} \alpha$ equivalent width listed by Cote \& Waters (1987). These were converted into line luminosities, assuming a black-body stellar continuum and using the tables in Straizys \& Kuriliene (1981) which list stellar radius and temperature vs. spectral type, to calculate the corresponding Planck function. As we can see in this paper, the Planck function is a poor approximation of the determination of the $\mathrm{H} \alpha$ luminosity. On the other hand, we needed to translate our fit along the $\log \left(L_{\mathrm{IR}}\right)$ axis, meaning that our IR luminosities were too faint, which is not surprising since our IR luminosities were calculated within a spectral bandwidth of only $40 \AA$. If we integrate our calculated IR fluxes at $2.16 \mu \mathrm{m}$ within a $22 \mu \mathrm{m}$ filter bandwith (mean value of the IRAS 12 and $25 \mu \mathrm{m}$ spectral bands), assuming that the calculated IR flux within the filter is constant, we obtain IR fluxes that 

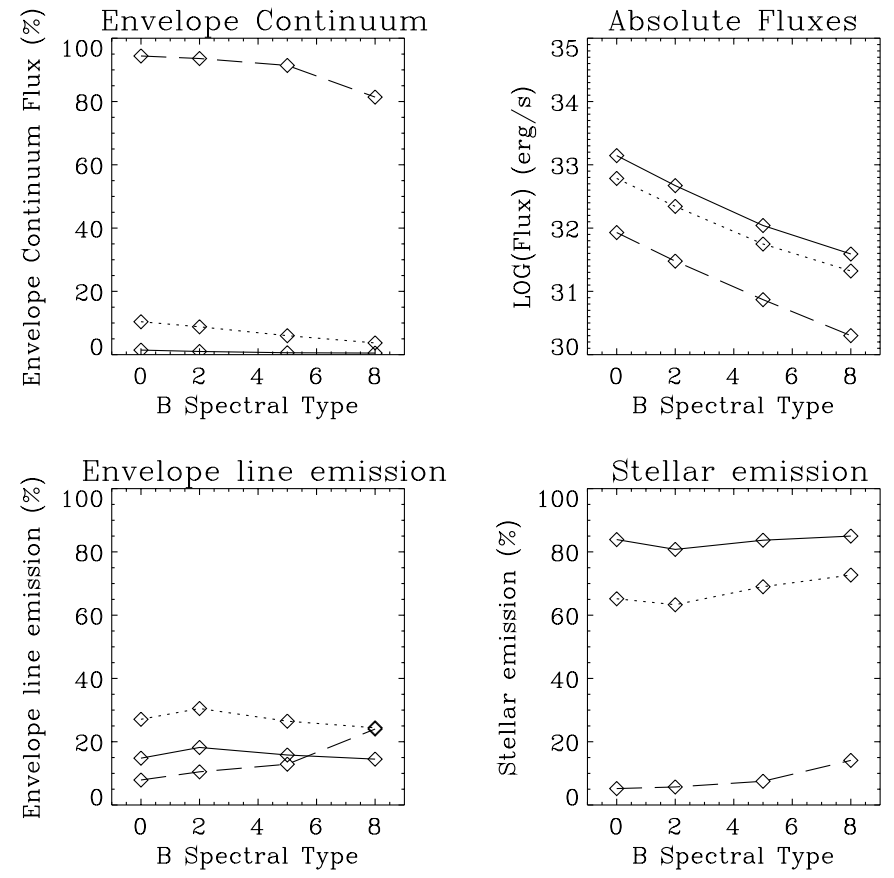

Fig. 13. Different flux contributions (in \%) as a function of the spectral type. For all plots we have the following convention: $\mathrm{H} \beta$ or $0.46 \mu \mathrm{m}$ (solid line), $\mathrm{H} \alpha$ or $0.65 \mu \mathrm{m}$ (dotted line), $\operatorname{Br} \gamma$ or $2.16 \mu \mathrm{m}$ (dashed line). Upper left: envelope continuum flux, Upper right: Log of the absolute fluxes in erg/s. Lower left: emission of the envelope in the line. Lower right: stellar contribution

Table 5. Stellar parameters for the different spectral types (from Zaal et al. 1995)

\begin{tabular}{|c|c|c|}
\hline Spectral type & Radius (in $R_{\odot}$ ) & $T_{\text {eff }}(\mathrm{K})$ \\
\hline \hline B0 & 6.0 & 30000 \\
\hline B2 & 4.3 & 23000 \\
\hline B5 & 3.0 & 15000 \\
\hline B8 & 2.5 & 12000 \\
\hline
\end{tabular}

are $4.2910^{35}, 1.4810^{35}, 3.5610^{34}$ and $8.2510^{33} \mathrm{erg} \mathrm{s}^{-1}$ respectively for the B0, B2, B5 and B8 spectral types. These values are in agreement with the determination of the IR luminosities from Kastner \& Mazzali (1989) and Chokshi \& Cohen (1987) based on IRAS data.

\subsection{Total flux of $B$ and Be stars versus spectral type}

In a paper by Briot et al. (1997), they present a study from Hipparcos data of the mean absolute magnitude as a function of spectral type for the B and Be stars. As was well known before, they found that the general trend for Be stars is an over luminosity compared to B stars of the same spectral type. A more interesting feature is that this over-luminosity is higher for later Be spectral types. In Fig. 16 we have plotted the ratio between the total flux from a B star and that of a Be star as a function of the spectral type. It is clear that for early-type stars this ratio is close to 1 , whereas it increases for later-type stars, i.e. the overluminosity increases with later Be spectral type, as found by Briot et al. (1997). This may be due to the fact that recombination in the envelope is less efficient for early-type stars but increases for later type stars which increases the overluminosity of late Be stars compared to "normal" B stars of the same spectral type.

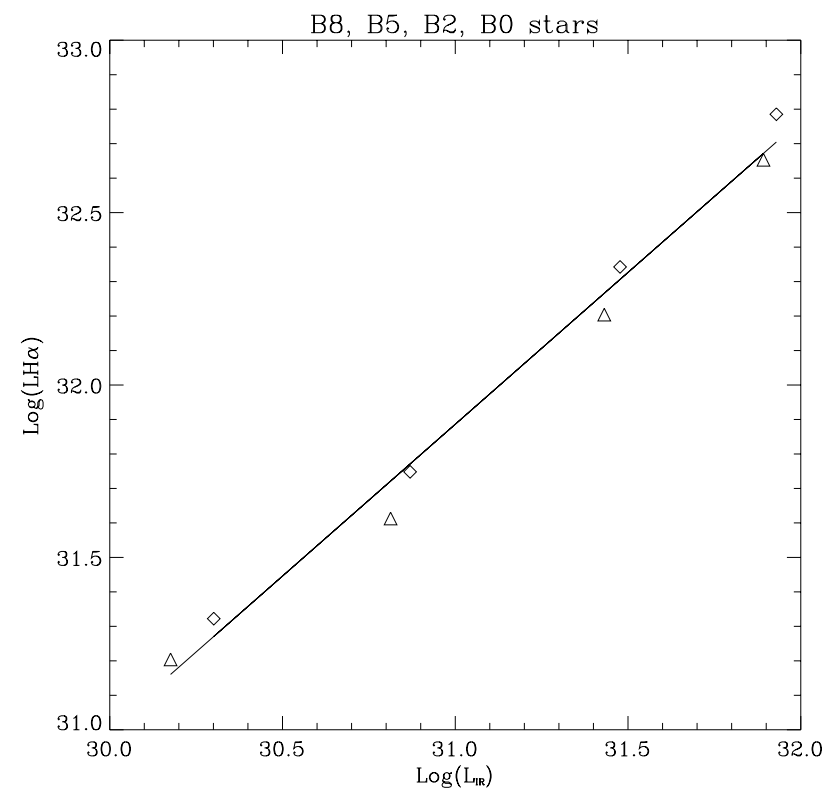

Fig. 14. Correlation $L_{\mathrm{H} \alpha}-L_{\mathrm{IR}}$ with the $\mathrm{B}$ spectral type. Diamonds indicate luminosity determination from the line $(\mathrm{H} \alpha$ and $\operatorname{Br} \gamma$ for the IR emission) whereas triangles indicate determination from the continua at $0.65 \mu \mathrm{m}$ and at $2.16 \mu \mathrm{m}$. All fluxes are taken within a spectral bandwidth of $40 \AA$. Spectral type decreases from the lower left to the upper right, i.e. from B8 to B0. Units are in $\log \left(\mathrm{erg} \mathrm{s}^{-1}\right)$

\section{Effect of the geometrical thickness of the disk}

Following the study by Stee (1998) who investigate the shape of the envelope around $\mathrm{B}[\mathrm{e}]$ supergiant stars, we have studied the effect of changing the geometrical thickness of the disk on the different flux contributions. Thus, we have computed different models with different $m 1$ parameters which describe the variation of the mass flux from the pole to the equator according to:

$\phi(\theta)=\phi_{\text {pole }}+\left[\left(\phi_{\text {eq. }}-\phi_{\text {pole }}\right) \sin ^{m 1}(\theta)\right]$

where $\theta$ is the stellar colatitude. The disk is very flat for $m 1=1000$ and forms a more or less ellipsoidal envelope for $m 1=0.1$. An example of meridian isodensity curves can be found in Fig. 3 of Stee (1998).

From Table 7, we can see that the envelope contribution in the line increases as the envelope becomes more and more ellipsoidal (lower $m 1$ ). At the same time, the stellar contribution decreases from a very flat envelope to a more ellipsoidal one due to an increase in the absorption of the stellar continuum from the circumstellar envelope. 
Table 6. Relative fluxes as a function of spectral type. The density at the base of the photosphere is constant and equal to $510^{-12} \mathrm{~g} \mathrm{~cm}^{-3}$

\begin{tabular}{|c|c|c|c|c|}
\hline & $\begin{array}{c}\text { Stellar contribution } \\
\text { (in \%) } \\
\end{array}$ & $\begin{array}{l}\text { Envelope contribution } \\
\text { in the line (in \%) } \\
\end{array}$ & $\begin{array}{c}\text { Envelope contribution } \\
\text { in the continuum (in \%) }\end{array}$ & $\begin{array}{c}\text { Total } \\
\text { (in } 10^{32} \mathrm{erg} \mathrm{s}^{-1} \text { ) } \\
\end{array}$ \\
\hline \multicolumn{5}{|l|}{ B0 } \\
\hline $\mathrm{H} \alpha$ & 65.23 & 27.17 & 7.6 & 6.1 \\
\hline $\mathrm{H} \beta$ & 83.92 & 14.84 & 1.23 & 14 \\
\hline $\mathrm{Br} \gamma$ & 5.2 & 7.95 & 86.85 & 0.85 \\
\hline $0.65 \mu \mathrm{m}$ & 89.57 & - & 10.43 & 4.5 \\
\hline $0.48 \mu \mathrm{m}$ & 98.55 & - & 1.45 & 12 \\
\hline $2.16 \mu \mathrm{m}$ & 5.61 & - & 94.39 & 0.78 \\
\hline \multicolumn{5}{|l|}{$\mathrm{B} 2$} \\
\hline $\mathrm{H} \alpha$ & 63.34 & 30.51 & 6.15 & 2.2 \\
\hline $\mathrm{H} \beta$ & 80.85 & 18.27 & 0.88 & 4.7 \\
\hline $\operatorname{Br} \gamma$ & 5.76 & 10.52 & 83.72 & 0.3 \\
\hline $0.65 \mu \mathrm{m}$ & 91.16 & - & 8.84 & 1.6 \\
\hline $0.48 \mu \mathrm{m}$ & 98.92 & - & 1.08 & 3.9 \\
\hline $2.16 \mu \mathrm{m}$ & 6.42 & - & 93.58 & 0.27 \\
\hline \multicolumn{5}{|l|}{ B5 } \\
\hline $\mathrm{H} \alpha$ & 69.07 & 26.52 & 4.42 & 0.56 \\
\hline $\mathrm{H} \beta$ & 83.70 & 15.82 & 0.48 & 1.1 \\
\hline $\mathrm{Br} \gamma$ & 7.53 & 12.90 & 79.57 & 0.074 \\
\hline $0.65 \mu \mathrm{m}$ & 93.99 & - & 6.01 & 0.41 \\
\hline $0.48 \mu \mathrm{m}$ & 99.43 & - & 0.57 & 0.94 \\
\hline $2.16 \mu \mathrm{m}$ & 8.63 & - & 91.37 & 0.065 \\
\hline \multicolumn{5}{|l|}{ B8 } \\
\hline $\mathrm{H} \alpha$ & 72.71 & 24.44 & 2.84 & 0.21 \\
\hline $\mathrm{H} \beta$ & 85.02 & 14.55 & 0.43 & 0.39 \\
\hline $\mathrm{Br} \gamma$ & 14.11 & 24.02 & 61.87 & 0.02 \\
\hline $0.65 \mu \mathrm{m}$ & 96.24 & - & 3.76 & 0.16 \\
\hline $0.48 \mu \mathrm{m}$ & 99.50 & - & 0.5 & 0.33 \\
\hline $2.16 \mu \mathrm{m}$ & 18.57 & - & 81.43 & 0.015 \\
\hline
\end{tabular}

Both effects tend to decrease the ratio of stellar/envelope continuum for more ellipsoidal geometries. Another interesting effect is that the envelope contribution in the $\mathrm{Br} \gamma$ line increases when the envelope becomes more "disk-like" contrary to the envelope contribution in the $\mathrm{H} \alpha$ and $\mathrm{H} \beta$ lines, which decreases. This is due to the increasing envelope continuum contribution for larger $m 1$ values which increases the ratio line/continuum for the $\operatorname{Br} \gamma$ line. The total flux decreases for flatter envelopes, i.e. for large $m 1$ values.

\section{Effect of the inclination angle}

Since the disk is optically thick in the lines, the line strength depends on the inclination angle. There are two main effects. (1) Since we solve the radiative transfer within the Sobolev approximation, the escape probability and thus the population of atomic levels strongly depends on the stellar latitude. The atomic populations rapidly decrease from the equator to the pole. (2) At the same time, the length of lines of sight crossing the disk increases and the optical depths become larger (which can be understood as a kind of "envelope-darkening" effect). From Table 8 we can directly measure these effects: (1) from the pole to the equator the stellar contribution decreases from
$80.6 \%$ to $38.1 \%$ for $\mathrm{H} \alpha$, i.e. it is more difficult to see the central star through the envelope, and (2) the envelope contribution in the lines increases with increasing inclination angle. Both effects tend to increase the strength of the lines with inclination angle.

Nevertheless, at the pole, the population of the levels decreases with distance much more rapidly, which produces a less important contribution at larger Doppler displacement and the polar profiles appear single peaked with a larger line strength. At the equator, it is the opposite: in our radiative wind model, the flow reaches its terminal velocity within a few stellar radii, a larger part of the envelope contributes a larger Doppler effect and the profiles appear double-peaked with a decreasing line strength. This is discussed in detail in Stee \& Araùjo (1994). Finally, we obtain the same trend for the effect of the inclination angle on the line profiles as those obtained by Marlborough et al. (1997) but from a completely different physical effect.

We have also studied the effect of the inclination angle on the visibilities. From Fig. 17 it is clear that the inclination angle has a large effect: for instance in $\mathrm{H} \alpha$, for the pole-on case (full line), the visibility is close to 0.8 for large baselines, whereas it falls down to 0.4 for the equator-on case (dashed line). Moreover, as it has been well shown 
Table 7. Relative fluxes as a function of the $m 1$ parameter. The density at the base of the photosphere is constant and equal to $510^{-12} \mathrm{~g} \mathrm{~cm}^{-3}$ and $T_{\text {eff }}=25000 \mathrm{~K}$

\begin{tabular}{|c|c|c|c|c|}
\hline & $\begin{array}{c}\text { Stellar contribution } \\
\text { (in \%) }\end{array}$ & $\begin{array}{l}\text { Envelope contribution } \\
\text { in the line (in \%) }\end{array}$ & $\begin{array}{l}\text { Envelope contribution } \\
\text { in the continuum (in \%) }\end{array}$ & $\begin{array}{c}\text { Total } \\
\left(\text { in } 10^{32} \mathrm{erg} \mathrm{s}^{-1}\right)\end{array}$ \\
\hline \multicolumn{5}{|l|}{$m 1=0.1$} \\
\hline $\mathrm{H} \alpha$ & 34.25 & 52.55 & 13.20 & 21 \\
\hline $\mathrm{H} \beta$ & 56.97 & 40.62 & 2.41 & 35 \\
\hline$\overline{\mathrm{Br} \gamma}$ & 1.52 & 7.59 & 90.89 & 5.6 \\
\hline $0.65 \mu \mathrm{m}$ & 72.17 & - & 27.83 & 10 \\
\hline $0.48 \mu \mathrm{m}$ & 95.94 & - & 4.06 & 21 \\
\hline $2.16 \mu \mathrm{m}$ & 1.62 & - & 98.38 & 5.2 \\
\hline \multicolumn{5}{|l|}{$m 1=6$} \\
\hline $\mathrm{H} \alpha$ & 40.72 & 47.94 & 11.34 & 20 \\
\hline$\overline{\mathrm{H} \beta}$ & 63.43 & 34.52 & 2.05 & 34 \\
\hline $\mathrm{Br} \gamma$ & 2.15 & 9.26 & 88.59 & 4.3 \\
\hline $0.65 \mu \mathrm{m}$ & 78.22 & - & 21.78 & 10 \\
\hline $0.48 \mu \mathrm{m}$ & 96.86 & - & 3.14 & 23 \\
\hline $2.16 \mu \mathrm{m}$ & 2.35 & - & 97.65 & 3.9 \\
\hline \multicolumn{5}{|l|}{$m 1=100$} \\
\hline $\mathrm{H} \alpha$ & 63.45 & 30.81 & 5.74 & 14 \\
\hline$\overline{\mathrm{H} \beta}$ & 81.19 & 17.82 & 0.99 & 31 \\
\hline$\overline{\mathrm{Br} \gamma}$ & 6.78 & 13.37 & 79.84 & 1.6 \\
\hline $0.65 \mu \mathrm{m}$ & 91.70 & - & 8.30 & 10 \\
\hline $0.48 \mu \mathrm{m}$ & 98.79 & - & 1.21 & 25 \\
\hline $2.16 \mu \mathrm{m}$ & 7.83 & - & 92.17 & 1.4 \\
\hline \multicolumn{5}{|l|}{$m 1=1000$} \\
\hline$\overline{\mathrm{H} \alpha}$ & 68.53 & 27.64 & 3.83 & 13 \\
\hline $\mathrm{H} \beta$ & 84.04 & 15.23 & 0.72 & 30 \\
\hline$\overline{\mathrm{Br} \gamma}$ & 11.31 & 18.36 & 70.33 & 0.95 \\
\hline $0.65 \mu \mathrm{m}$ & 94.71 & - & 5.29 & 9.6 \\
\hline $0.48 \mu \mathrm{m}$ & 99.15 & - & 0.85 & 25 \\
\hline $2.16 \mu \mathrm{m}$ & 13.85 & - & 86.15 & 0.77 \\
\hline
\end{tabular}

using the MkIII interferometer (Quirrenbach et al. 1997), the envelope geometry of Be stars is not only dependent on the inclination angle but also on the orientation of the disk elongation into the sky plane, which is another important free parameter that can be constrained only by spectropolarimetry or interferometry. For instance, thanks to simultaneous optical interferometry and spectropolarimetry measurements, Quirrenbach et al. (1997) have found that the polarization angles were perpendicular to the interferometric major axii for seven Be stars and favors disk geometry over mildly ellipsoidal models. Thus, we require simultaneously high spectral resolution as well as high angular resolution observations in order to fully constrain the free parameters of Be star models.

\section{Effect of rotational and terminal wind velocity}

Since the $\operatorname{Br} \gamma$ line originates from very extended regions it may be sensitive to the rotational velocity field which takes place in the inner region but also to the slowly expanding equatorial velocity which reaches its terminal velocity in a few stellar radii. In order to check this effect, we have computed $\operatorname{Br} \gamma$ lines for 3 different rotational velocities, i.e. 325,300 and $250 \mathrm{~km} \mathrm{~s}^{-1}$. From Fig. 18 we can see that the FWHM of the $\mathrm{Br} \gamma$ line profile is independent of the equatorial rotational velocity. The only difference appears in the intensity of the red $(R)$ and violet $(V)$ peaks of the profile, which are smaller for lower velocities. On the contrary Fig. 19 shows clearly that $\mathrm{Br} \gamma$ is very sensitive to an equatorial terminal velocity change. Both intensity and FWHM of the profiles decrease with decreasing terminal velocity.

If we compare our FWHM for the $\mathrm{Br} \gamma$ line with the value of $280 \mathrm{~km} \mathrm{~s}^{-1}$ obtained by Chalabaev \& Maillard (1985) for $\gamma$ Cas in 1982, we find that a terminal velocity of $\sim 100 \mathrm{~km} \mathrm{~s}^{-1}$ is needed to fit their data. This is in agreement with the value of $90 \mathrm{~km} \mathrm{~s}^{-1}$ they obtained for the velocity of the outflow. Nevertheless, they also interpret the quasi-symmetric $\operatorname{Br} \gamma$ profile (with $V / R \sim 0.85$ ) as direct evidence of an inner $\left(R<3 R_{*}\right)$ region where rotation dominates. In our model, we have the same geometry, i.e. an inner region dominated by the rotation of the envelope and outer layers dominated by expansion. Nevertheless, in our case, $\operatorname{Br} \gamma$ originates from extended regions $\left(1 R_{*}<R<20 R_{*}\right)$ and the profile presents a $V / R$ ratio $\sim 0.90$ for a terminal velocity of $100 \mathrm{~km} \mathrm{~s}^{-1}$, which is very close to the $V / R \sim 0.85$ they obtained. Thus, even if $\mathrm{Br} \gamma$ is formed in an extended region, it is also 
Table 8. Relative fluxes as a function of the inclination angle $(i)$. The density at the base of the photosphere is constant and equal to $510^{-12} \mathrm{~g} \mathrm{~cm}^{-3}$ and $T_{\text {eff }}=25000 \mathrm{~K}$

\begin{tabular}{|c|c|c|c|c|}
\hline & $\begin{array}{c}\text { Stellar contribution } \\
\text { (in \%) }\end{array}$ & $\begin{array}{l}\text { Envelope contribution } \\
\text { in the line (in \%) }\end{array}$ & $\begin{array}{c}\text { Envelope contribution } \\
\text { in the continuum (in \%) }\end{array}$ & $\begin{array}{c}\text { Total } \\
\left(\text { in } 10^{32} \mathrm{erg} \mathrm{s}^{-1}\right)\end{array}$ \\
\hline \multicolumn{5}{|c|}{$\bar{i} i=0^{\circ}($ pole-on $)$} \\
\hline $\mathrm{H} \alpha$ & 80.63 & 15.61 & 3.76 & 60 \\
\hline $\mathrm{H} \beta$ & 91.33 & 8.17 & 0.50 & 150 \\
\hline$\overline{\mathrm{Br} \gamma}$ & 11.76 & 8.08 & 80.16 & 4.8 \\
\hline $0.65 \mu \mathrm{m}$ & 95.54 & - & 4.46 & 51 \\
\hline $0.48 \mu \mathrm{m}$ & 99.45 & - & 0.55 & 130 \\
\hline $2.16 \mu \mathrm{m}$ & 12.80 & - & 87.20 & 4.4 \\
\hline \multicolumn{5}{|l|}{$i=45^{\circ}$} \\
\hline $\mathrm{H} \alpha$ & 56.94 & 35.94 & 7.12 & 26 \\
\hline$\overline{\mathrm{H} \beta}$ & 76.80 & 22.06 & 1.12 & 52 \\
\hline $\operatorname{Br} \gamma$ & 4.59 & 10.54 & 84.87 & 3.7 \\
\hline $0.65 \mu \mathrm{m}$ & 88.89 & - & 11.11 & 16 \\
\hline $0.48 \mu \mathrm{m}$ & 98.56 & - & 1.44 & 41 \\
\hline $2.16 \mu \mathrm{m}$ & 5.07 & - & 94.93 & 3.3 \\
\hline \multicolumn{5}{|c|}{$i=90^{\circ}$ (equator-on) } \\
\hline $\mathrm{H} \alpha$ & 38.10 & 53.32 & 9.57 & 17 \\
\hline $\mathrm{H} \beta$ & 60.18 & 38.16 & 1.66 & 30 \\
\hline $\mathrm{Br} \gamma$ & 2.21 & 12.32 & 85.47 & 3.5 \\
\hline $0.65 \mu \mathrm{m}$ & 79.92 & - & 20.08 & 8.3 \\
\hline $0.48 \mu \mathrm{m}$ & 97.31 & - & 2.69 & 19 \\
\hline $2.16 \mu \mathrm{m}$ & 2.46 & - & 97.54 & 3.0 \\
\hline
\end{tabular}

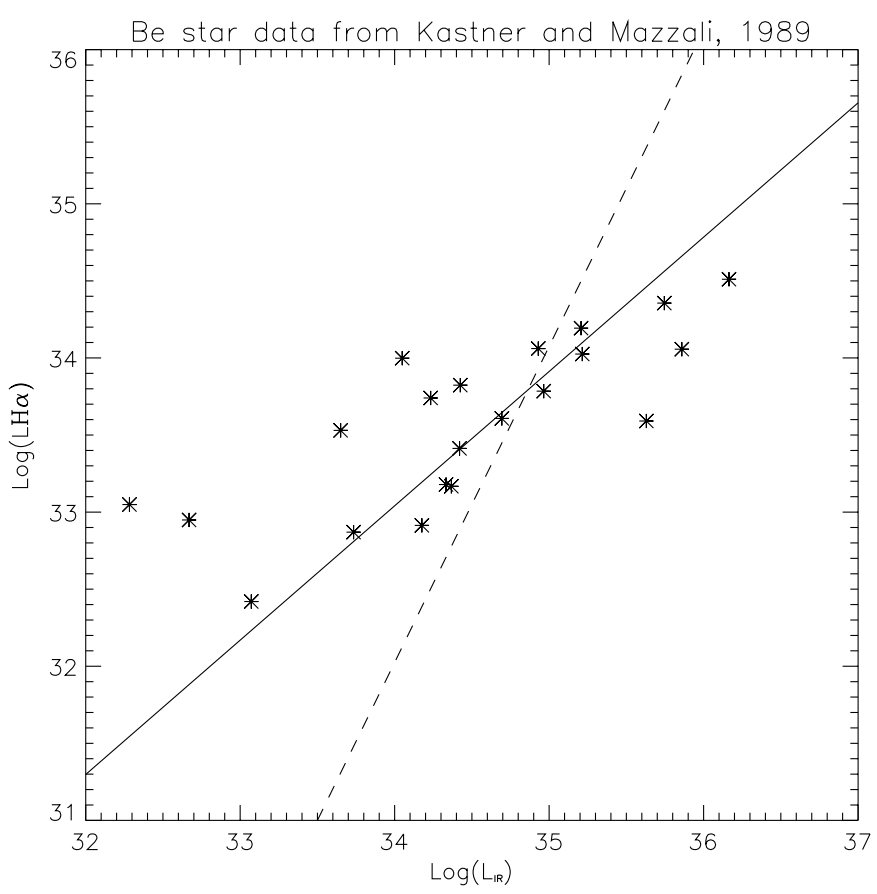

Fig. 15. $L_{\mathrm{H} \alpha}-L_{\mathrm{IR}}$ data from Kastner \& Mazzali (1989) using a sample of 21 Be stars. The full line is a fit using Eq. (3) were we have translated the $\log (\mathrm{H} \alpha)$ axis by 1.46 . The dashed line is from the correlation obtained as a function of the temperature using Eq. (2)

possible to obtain a FWHM and a $V / R$ ratio that fits their observational data.

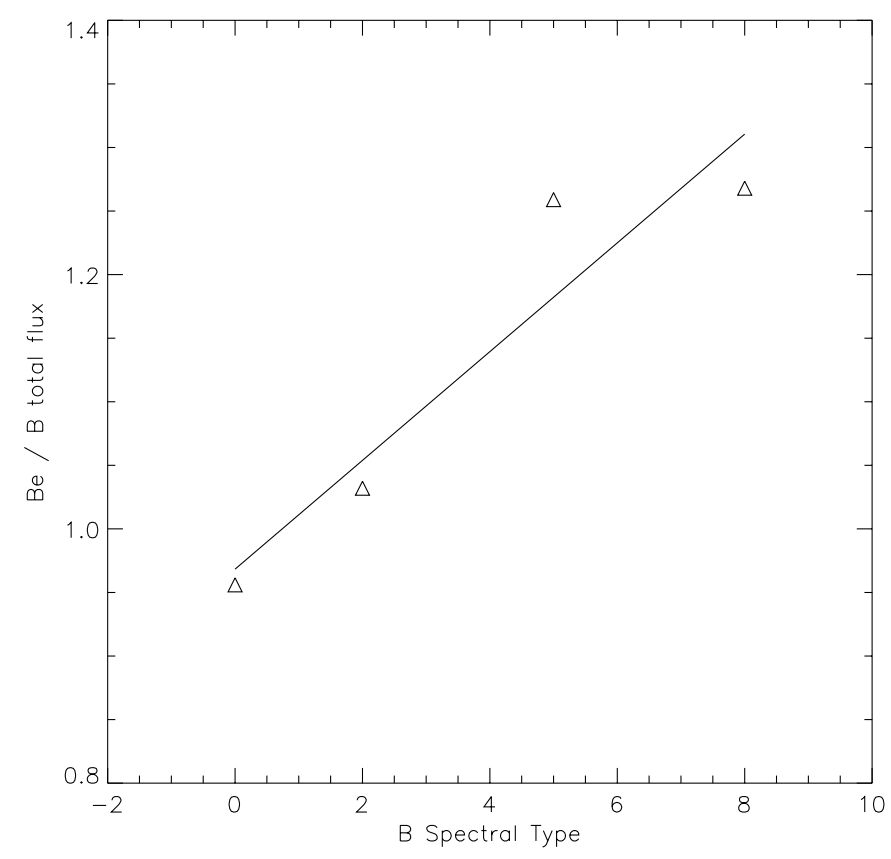

Fig. 16. Be/B total flux ratio as a function of the B spectral type

\section{Conclusion and discussion}

Using the SIMECA code developed by Stee et al. (1994), (1995) we have carried out a study of theoretical HI visible, $\mathrm{H} \alpha, \mathrm{H} \beta$, and near-IR $\mathrm{Br} \gamma$ line profiles for a large set of parameters (density, temperature, envelope oblateness, 

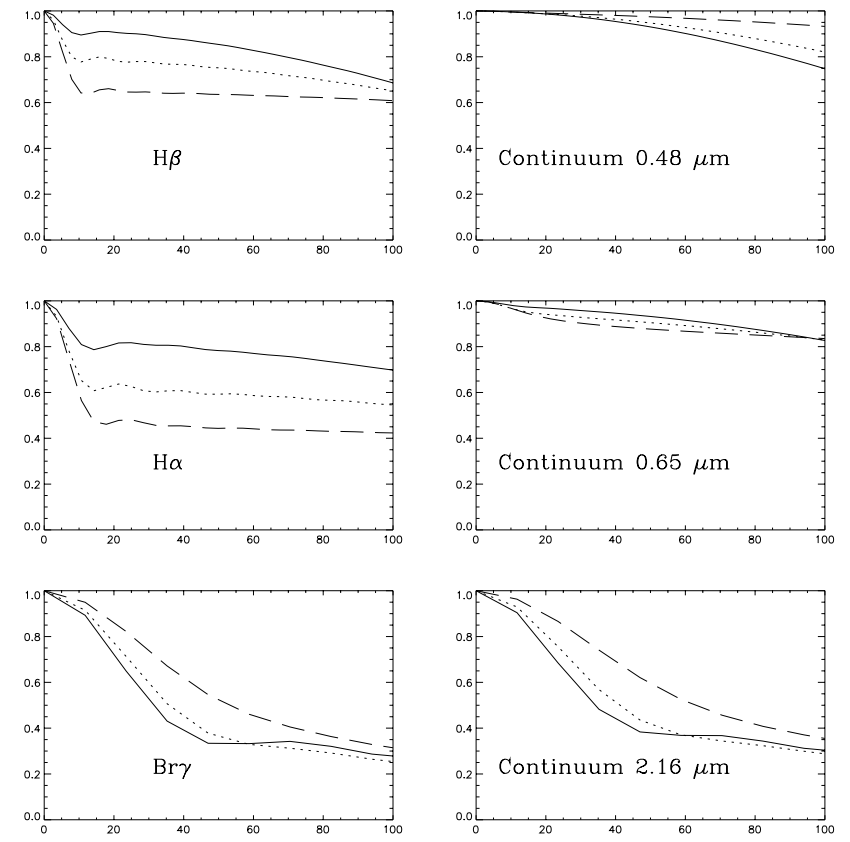

Fig. 17. Visibilities as a function of the inclination angle. Full line: pole-on, dotted line: $i=45^{\circ}$, dashed line: equator-on. Baselines are between 0 and 100 meters. Wavelengths or lines are indicated on each plot

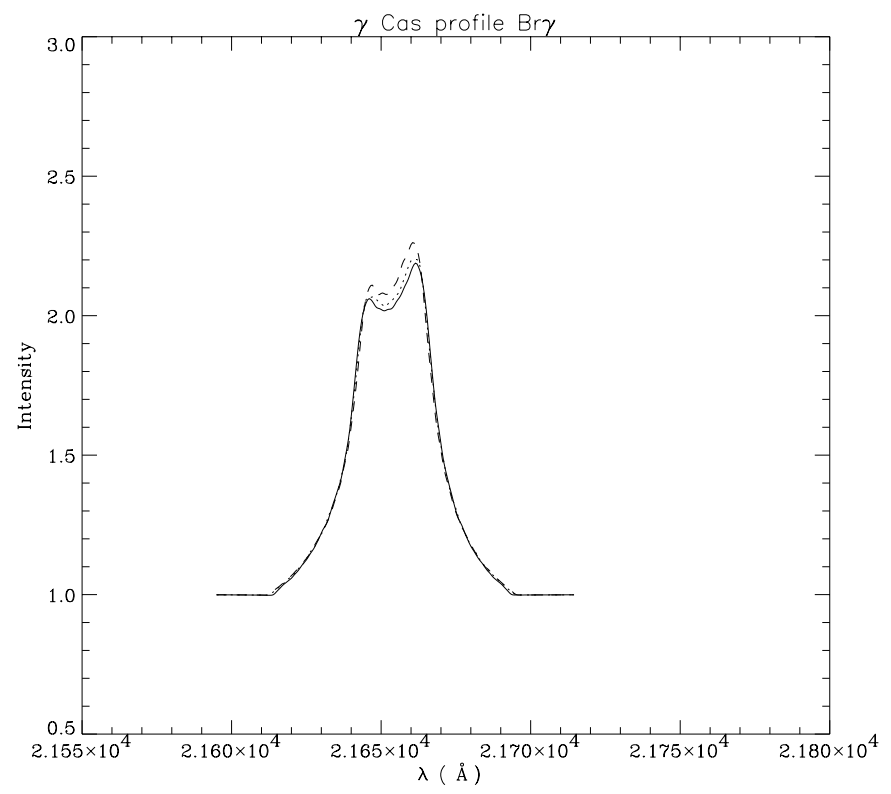

Fig. 18. Br $\gamma$ line profile for different equatorial rotational velocities: $325 \mathrm{~km} \mathrm{~s}^{-1}$ (solid line), $300 \mathrm{~km} \mathrm{~s}^{-1}$ (dotted line) and $250 \mathrm{~km} \mathrm{~s}^{-1}$ (dashed line)

inclination angle). We have also computed energy distribution, intensity maps and corresponding visibility curves which allow us to draw the following conclusions:

- The near-IR emission both in the $\operatorname{Br} \gamma$ line and the nearby continuum originates from a very extended region with a typical size of 40 stellar radii which is twice the size of the $\mathrm{H} \alpha$-emitting region. This prediction may be compared to previous estimates of the disk extension at various wavelengths, compiled in Table 9.

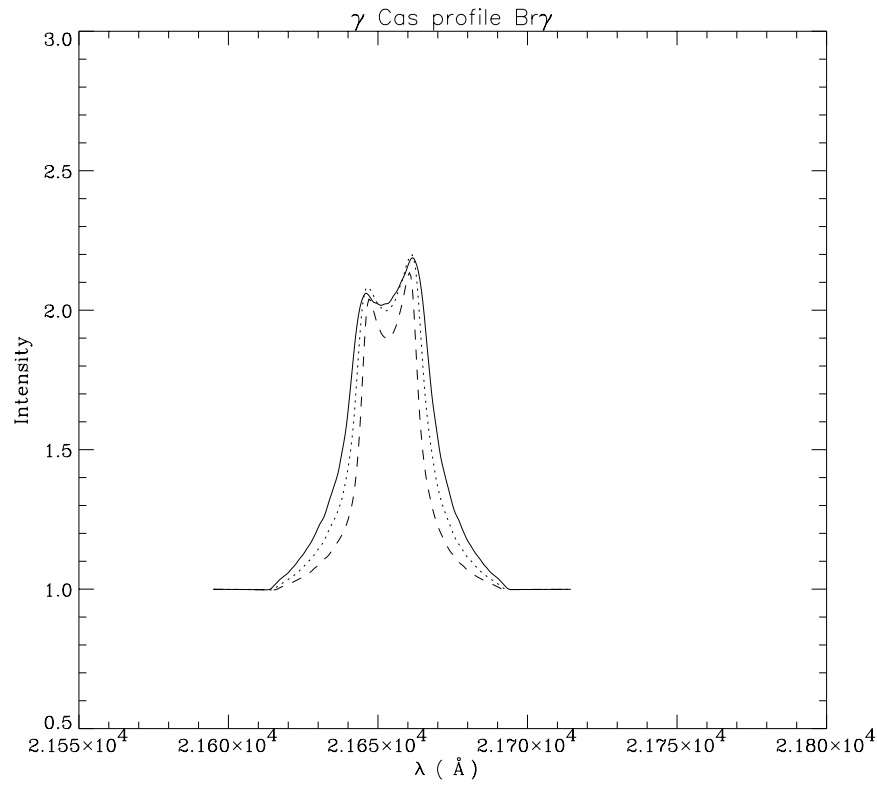

Fig. 19. Br $\gamma$ line profile for different terminal velocities: $200 \mathrm{~km} \mathrm{~s}^{-1}$ (solid line), $150 \mathrm{kms}^{-1}$ (dotted line) and $100 \mathrm{~km} \mathrm{~s}^{-1}$ (dashed line). The corresponding FWHM are respectively 401,339 and $277 \mathrm{~km} \mathrm{~s}^{-1}$

We must mention that all these measurements are model-dependent and sometimes solutions with an "infinite" disk size can be found (see for instance Waters et al. 1987). In fact, our large near-IR extension is mainly due to the lower brightness contrast between the central star and the envelope. In the visible the star appears very bright and thus it is more difficult to "see" the circumstellar matter. In the near-IR, the central star is very faint and the surrounding matter can be seen at very large distances. It is clear that first interferometric measurements of Be stars at near-IR wavelengths will definitely favor one of the given extensions even if details of the disk structure itself will require more refined models.

- We have computed the envelope size of the Be star $\gamma$ Cas in the $\mathrm{H} \alpha, \mathrm{H} \beta, \mathrm{Br} \gamma$ lines and at 0.65, 0.48 and $2.16 \mu \mathrm{m}$ in the continuum. In the He I $\lambda 6678$ line our previous study predicted a size of $2.3 R_{*}$, due to the fact that we had substracted the nearby continuum in the corresponding intensity map. Taking into account the continuum emitted from the envelope, we obtain a size of $\sim 4 R_{*}$ in better agreement with the $3.7 R_{*}$ obtained at $0.65 \mu \mathrm{m}$.

- We have predicted the relative fluxes from the central star, the envelope contribution in the $\mathrm{H} \alpha, \mathrm{H} \beta, \mathrm{Br} \gamma$ lines and in the continuum as well as the total fluxes in erg $\mathrm{s}^{-1}$, for a wide range of parameters characterizing the disk models. For the same parameters, we have also predicted the sensitivity of the lines, the energy distribution and the visibilites in the lines and in the continuum. The density at the base of the photosphere and the inclination angles are the most sensitive parameters and a factor of 2 or 3 may be found between the different fluxes from the extreme cases. We found that for densities $\sim 510^{-11} \mathrm{~g} \mathrm{~cm}^{-3}$, the stellar flux at $2.16 \mu \mathrm{m}$ is 
Table 9. Estimation of the disk size from various authors as a function of wavelength

\begin{tabular}{|c|c|c|}
\hline Wavelength & Diameter (in stellar radii) & Reference \\
\hline \hline 2.3 and $19.5 \mu \mathrm{m}$ & $8 R_{*}$ & Gehrz et al. (1974) \\
\hline 12,25 and $60 \mu \mathrm{m}$ & $16 R_{*}$ & Waters (1986) \\
\hline IRAS Far-IR & $8<\phi_{\text {disk }}<26 R_{*}$ & Waters et al. (1987) \\
\hline Millimeter & $52<\phi_{\text {disk }}<216 R_{*}$ & Waters et al. (1991) \\
\hline near-IR & $\phi_{\text {disk }}>20 R_{*}$ & Dougherty et al. (1994) \\
\hline $2 \mathrm{~cm}$ & $\phi_{\text {disk }}>200 R_{*}$ & Taylor et al. (1990) \\
\hline
\end{tabular}

only about $1 \%$ which is much smaller than the $20 \%$ obtained from Hony et al. (2000) with the same density but from a Kurucz model atmosphere extrapolation fitted to the UV continuum. In SIMECA our final atomic levels are strongly NLTE-distributed, as found by Hony et al. (2000) from the study of the infrared spectrum of $\gamma$ Cas. For $\rho=$ $510^{-13} \mathrm{~g} \mathrm{~cm}^{-3}$ we have obtained the largest probability of having HI IR lines in emission, which is a factor of 100 lower than the typical values for Be stars and close to the value $\rho \sim 10^{-14} \mathrm{~g} \mathrm{~cm}^{-3}$ found by Zaal et al. (1995).

- We have studied the effect of spectral type on our results and we obtain a good correlation between the luminosity in $\mathrm{H} \alpha$ and in the infrared, as found by Neto \& de Freitas Pacheco (1982) and Kastner \& Mazzali (1989). We have also found that, for $\rho=510^{-12} \mathrm{~g} \mathrm{~cm}^{-3}$, the probability of detecting HI IR lines in emission is stronger for normal late B type stars contrary to Zaal et al. (1995) who obtain the reverse, i.e. a stronger probability for early type stars with low density disks. If no IR lines are detected for late B spectral types it may be an indication that:

a) the density in the disc is very low $\left(\sim 10^{-14} \mathrm{~g} \mathrm{~cm}^{-3}\right)$ and near-IR emission lines are not detected but in this case the density is also too low to produce Balmer lines in emission;

b) the density in the disc is very high $\left(\sim 10^{-11} \mathrm{~g} \mathrm{~cm}^{-3}\right)$ and due to the strong underlying continuum from the envelope, the line to continuum ratio is smaller in the nearIR lines, whereas Balmer lines are still visible.

- On the otherhand, we also found that around a density of $510^{-13} \mathrm{~g} \mathrm{~cm}^{-3}$ it is possible to have an important envelope contribution in the $\mathrm{Br} \gamma$ line and a similar or even smaller emission in the Balmer lines. This density is close to that needed to model the B0.2V star $\tau$ Sco spectrum in order to obtain strong IR HI emission lines without noticeable emission in the photospheric $\mathrm{H} \alpha$ absorption line (Zaal et al. 1995).

- As found by Briot et al. (1997) we obtain that the overluminosity of Be stars as compared with "normal" B stars of the same spectral type increases with later Be spectral types, which may be due to the fact that recombination in the envelope is less efficient for early-type stars whereas it increases for later-type stars.

- The equatorial rotational velocity can slightly modify the intensity of the $\mathrm{Br} \gamma$ profile, which is smaller for lower velocities. $\operatorname{Br} \gamma$ itself is very sensitive to an equatorial terminal velocity change. Both intensity and FWHM of the profiles decrease with decreasing terminal velocity.
- Finally, it seems that the contribution in the $\operatorname{Br} \gamma$ line increases when the envelope becomes more "disk-like", in contrast to the $\mathrm{H} \alpha$ and $\mathrm{H} \beta$ lines.

In conclusion, it is clear that the forthcoming VLT interferometer and its near-IR focal instrument AMBER (Richichi et al. 2000) and the GI2T/REGAIN interferometer working in dispersed fringe mode at near-IR wavelengths (Weigelt et al. 2000) will settle the question of whether the IR-emiting region is extended, which will provide strong constraints on the physics of Be stars.

We also hope to present in the near future new results from a modified version of the SIMECA code without the Sobolev approximation but with a fully $3 \mathrm{D}$ radiative transfer and which will integrate the "one-armed" oscillations phenomenon (Okazaki 1991) in order to reproduce the observed long term variability.

Acknowledgements. The authors thank C. Thomas and T. Girard for their help in the preparation of the figures presented in this paper. We thank A.-M. Hubert, J. Zorec, D. Briot and J. Pacheco for many helpful suggestions and careful reading of the manuscript. The useful remarks of the referee R. MillanGabet are also greatly acknowledged by the authors. Ph. Stee acknowledges the Ministère de la Recherche and the operation "Coup de Pouce Jeunes Chercheurs" for his financial support.

\section{References}

Briot, D., Robichon, N., \& Hubert, A. M. 1997, Proceeding of ESA Symposium "Hipparcos - Venice 97", 13-16 May, Venice, Italy, ESA SP-402, 319

Castor, J. I., Abbott, D. C., \& Klein, R. I. 1975, ApJ, 195, 157

Chokshi, A., \& Cohen, M. 1987, AJ, 94, 123

Chalabaev, A. A., \& Maillard, J. P. 1982, ApJ, 294, 640

Clark, J. S., \& Steele, I. A. 2000, A\&AS, 141, 65

Cote, J., \& Waters, L. B. F. M. 1987, A\&A, 176, 93

Dachs, J., Engels, D., \& Kiehling, R. 1988, A\&A, 194, 167

Dougherty, S. M., Waters, L. B. F. M., Burki, G., et al. 1994, A\&A, 290, 609

Gehrz, R. D., Hackwell, J. A., \& Jones, T. W. 1974, ApJ, 191, 675

Hamann, F., \& Simon, M. 1987, ApJ, 318, 356

Hony, S., Waters, L. B. F. M., Zaal, P. A., et al. 2000, A\&A, 355,187

Hubeny, I. 1988, Comp. Phys. Comm., 52, 103

Hubeny, I., \& Lanz, T. 1995, ApJ, 439, 875

Kastner, J. H., \& Mazzali, P. A. 1989, A\&A, 210, 295

Marlborough, J. M., Zijlstra, J.-W., \& Waters, L. B. F. M. 1997, A\&A, 321, 867

Neto Augusto Damineli, \& de Freitas Pacheco, J. A. 1982, MNRAS, 198, 659 
Okazaki, A. T. 1991, PASJ, 43, 75

Quirrenbach, A., Hummel, C. A., Buscher, D. F., et al. 1993, ApJ, 416, L25

Quirrenbach, A., Buscher, D. F., Mozurkewich, D., et al. 1994, A\&A, 283, L13

Quirrenbach, A., Bjorkman, K. S., Bjorkman, J. E., et al. 1997, ApJ, 479, 477

Richichi, A., Blöcker, T., Foy, R., et al. 2000, SPIE "Interferometry in Optical Astronomy" Proc. Ser., vol. 4006, Part I, 80

Stee, Ph., \& de Araùjo, F. X. 1994, A\&A, 292, 221

Stee, Ph., de Araùjo, F. X., Vakili, F., et al. 1995, A\&A, 300, 219

Stee, Ph. 1996, A\&A, 311, 945

Stee, Ph., Vakili, F., Bonneau, D., et al. 1998, A\&A, 332, 268

Stee, Ph. 1998, A\&A, 336, 980

Stee, Ph. 2000, The Be phenomenon in Early-Type stars, ed. M. A., Smith, H. F., Henrichs, \& J. Fabregat, PASP Conf. Ser., 214, 129

Struve, O. 1931, ApJ, 73, 94

Straizys, V., \& Kuriliene, G. 1981, Astrophys. Space Sci., 80, 353
Taylor, A. R., Waters, L. B. F. M., \& Bjorkman, K. S. 1990, A\&A, 231, 453

Telting, J. H., \& Kapler, L. 1994, A\&A, 284, 515

Telting, J .H., Waters, L. B. F. M., Persi, P., et al. 1993, A\&A, 270,355

Tuthill, P. G., Monnier, J. D., Danchi, W. C., et al. 2000, PASP, 112,555

Thom, C., Granes, P., \& Vakili, F. 1986, A\&A, 165, L13

Vakili, F., Mourard, D., Bonneau, D., et al. 1997, A\&A, 323, 183

Vakili, F., Mourard, D., Stee, Ph., et al. 1998, A\&A, 335, 261

Waters, L. B. F. M. 1986, A\&A, 162, 121

Waters, L. B. F. M., Coté, J., \& Lamers, H. J. G. L. M. 1987, A\&A, 185, 206

Waters, L. B. F. M., van der Veen, W. E. C. J., Taylor, A. R., et al. 1991, A\&A, 244, 120

Weigelt, G., Mourard, D., Abe, L., et al. 2000, SPIE "Interferometry in Optical Astronomy" Proc. Ser., vol. 4006, Part II, 617

Zaal, P. A., Waters, L. B. F. M., \& Marlborough, J. M. 1995, A\&A, 299, 574 